\title{
Una visión desde la red de teorías TAC-EOS sobre el papel de las conexiones matemáticas en la comprensión de la derivada
}

\author{
Camilo Andrés Rodríguez-Nieto \\ Flor Monserrat Rodríguez-Vásquez \\ Vicenç Font Moll \\ Armando Morales-Carballo
}

\begin{abstract}
Resumen: Se responde a la pregunta ¿Dada la expresión algebraica de $g$, qué conexiones aseguran al estudiante una comprensión de $g^{\prime}$ que le permite esbozar la gráfica de $g$ y explicar la relación que tiene con la de $g^{\prime}$ ? Para ello, a un grupo de estudiantes se les aplicaron entrevistas y una tarea, en la que debían esbozar la gráfica de $g$ y explicar la relación que tiene con la de la derivada, y se analizó su actividad matemática usando como referente teórico la articulación TAC-EOS. Los estudiantes que evidenciaron una comprensión que les permitió resolver la tarea establecieron conexiones de: representaciones diferentes, significado, parte-todo, implicación y característica. Por otra parte, el no establecimiento de determinadas conexiones es una explicación plausible de por qué algunos estudiantes no resuelven la tarea.
\end{abstract}

Camilo Andrés Rodríguez-Nieto Doctor en Ciencias con Especialidad en Matemática Educativa por la Universidad Autónoma de Guerrero (UAGro), México. Profesor catedrático en la Universidad del Atlántico (UA), Colombia. Coordinador de Semillero de investigación Conexiones Etnomatemáticas, Teóricas y Metodológicas en Educación Matemática (CETMEM) adscrito al Grupo de Investigación Horizontes en Educación Matemática (GIHEM) de la UA

http://orcid.org/0000-0001-9922-4079 camiloarodriguez@mail.uniatlantico.ed u.CO

Flor Monserrat Rodríguez-Vásquez Doctora en Educación Matemática. Profesora en el programa de Doctorado y maestría en Matemática Educativa de la Universidad Autónoma de Guerrero, Chilpancingo, Guerrero (UAGro), México.

iD http://orcid.org/0000-0002-9596-4253 $\triangle$ flor.rodriguez@uagro.mx

Vicenç Font Mol Doctor en filosofía y ciencias de la Educación. Profesor en el programa de Doctorado en Didáctica de las Ciencias, las Lenguas, las Artes y las Humanidades de la Universitat de Barcelona (UB), España.

(D) http://orcid.org/0000-0003-1405-0458 $\triangle$ vfont@ub.edu

Armando Morales-Carballo Doctor en Ciencias con Especialidad en Matemática Educativa. Profesor titular de

la Facultad de Matemáticas de la

Universidad Autónoma de Guerrero (UAGro), México

http://orcid.org/0000-0001-9841-7493

$\bowtie$ armandomorales@uagro.mx

Recebido em 21/06/2021

Aceito em 17/07/2021

Publicado em 24/07/2021
Palabras clave: Comprensión. Teoría Ampliada de las Conexiones. Enfoque Ontosemiótico. Derivada. Gráfica.

\section{Uma visão da rede de teorias TAC-EOS sobre o papel das conexões matemáticas na compreensão da derivada}

Resumo: A pergunta é respondida: Dada a expressão algébrica de $\boldsymbol{g}$, que conexões garantem ao aluno uma compreensão de $\boldsymbol{g}^{\prime}$ que lhe permite esboçar o gráfico de $\boldsymbol{g}$ e explicar sua relação com o de $\boldsymbol{g}^{\prime}$ ? Para isso, foram aplicadas entrevistas e uma tarefa a um grupo de alunos, em que eles tiveram que esboçar o gráfico de $\boldsymbol{g}$ e explicar sua relação com o da derivada, e sua atividade matemática foi analisada usando a articulação TAC-EOS como um ponto teórico referência. Os alunos que mostraram uma compreensão que lhes permitiu resolver a tarefa estabeleceram conexões de: diferentes representações, significado, parte-todo, implicação e característica. Por outro lado, a falha em estabelecer certas conexões é uma explicação plausível porque alguns alunos não resolvem a tarefa.

Palavras-chave: Compreensão. Teoria Expandida das Conexões. Enfoque ontossemiótico. Derivada. Gráfico.

\section{A view from the ETC-OSA networking of theories on the role of mathematical connections in understanding the derivative}

Abstract: The question is answered: Given the algebraic expression of $g$, what connections ensure the student an understanding of $g^{\prime}$ that allows him to sketch the graph of $g$ and explain its relationship with that of $g^{\prime}$ ? To do this, interviews and a task were applied to a group of students, in which they had to sketch the graph of $g$ and explain its relationship with that of the derivative, and their mathematical activity was analyzed using the ETC-OSA articulation as a theoretical reference. The students who showed an understanding that allowed them to solve the task 
established connections of: different representations, meaning, part-whole, implication and feature. On the other hand, the failure to establish certain connections is a plausible explanation for why some students do not solve the task.

Keywords: Understanding. Extended Theory of Connections. Onto-semiotic Approach. Derivative. Graph.

\title{
1 Introducción
}

El establecimiento de conexiones matemáticas es importante para que los estudiantes comprendan conceptos matemáticos, relacionen significados, representaciones, usen fórmulas adecuadamente para hacer procedimientos y vinculen las matemáticas con situaciones de la vida real (NATIONAL COUNCIL OF TEACHERS OF MATHEMATICS [NCTM], 2000). La investigación en Educación Matemática reconoce que las conexiones son un indicador de comprensión y esta viene dada por una red de conexiones (BERRY y NYMAN, 2003; CAI y DING, 2015; HIEBERT y CARPENTER, 1992; RODRÍGUEZ-NIETO, 2020; 2021; RODRÍGUEZ-VÁSQUEZ y ARENAS, 2021). En este contexto, Kenedi et al. (2019) afirmaron que

\begin{abstract}
La capacidad de los estudiantes para conectar ideas matemáticas es una de las cosas esenciales que deben lograr los estudiantes en el proceso de aprendizaje porque si los estudiantes conocen la relación entre los conceptos, comprenderán rápidamente las matemáticas en sí y abrirán oportunidades para que los estudiantes desarrollen sus habilidades matemáticas. (p. 70)
\end{abstract}

De hecho, las conexiones matemáticas se relacionan con la resolución de problemas matemáticos, al respecto, Pambudi, Budayasa y Lukito (2018) sostienen que "la capacidad de los estudiantes para conectar ideas matemáticas determinará el éxito de los estudiantes en la resolución de problemas matemáticos" (p. 74). Por su parte, Font (2007) afirma que, "un sujeto comprende un determinado objeto matemático cuando lo usa de manera competente en diferentes prácticas" (p. 107), en la resolución de problemas intra o extramatemáticos.

Sobre las conexiones matemáticas se ha investigado ampliamente, destacándose estudios centrados en las conexiones que hacen estudiantes de nivel primaria (KENEDI et al., 2019), estudios que han identificado las conexiones de estudiantes del preuniversitarios en el contexto de la derivada y la integral (GARCÍA-GARCÍA y DOLORES-FLORES, 2018; 2019; 2020) y conexiones en la resolución de problemas sobre la razón de cambio (DOLORES-FLORES, RIVERA-LÓPEZ y GARCÍA-GARCÍA, 2019). En el nivel universitario, algunas investigaciones muestran que los estudiantes de licenciatura en matemáticas hacen conexiones intramatemáticas sobre la derivada especialmente entre significados (pendiente de la recta tangente a la curva en un punto y el límite del cociente de las tasas medias de variación de la función), pero también conexiones de otros tipos: entre representaciones diferentes, metafórica, característica, parte-todo 
e implicación (RODRÍGUEZ-NIETO, FONT, BORJI y RODRÍGUEZ-VÁSQUEZ, 2021), y conexiones extramatemáticas en problemas de Cálculo (DOLORES-FLORES y GARCÍA-GARCÍA, 2017).

Otros estudios se han enfocado en las conexiones que hacen futuros profesores de matemáticas (RODRÍGUEZ-NIETO, RODRÍGUEZ-VÁSQUEZ y GARCÍA-GARCÍA, 2021; YAVUZMUMCU, 2018), especialmente en Rodríguez-Nieto et al. (2021) algunos futuros profesores presentaron algunas dificultades para hallar la ecuación de la recta tangente, producto del significado inadecuado que tienen de la derivada. En el contexto de los profesores en servicio, Businskas (2008) analizó las conexiones realizadas por profesores de secundaria en la resolución de tareas que implican a la ecuación y función cuadrática. Asimismo, en los trabajos de Mhlolo (2012) y Mhlolo, Venkat y Schäfer (2012) se indagó acerca de la calidad de las conexiones matemáticas que hacen profesores, enfocados especialmente en las conexiones de representaciones diferentes. Rodríguez-Nieto, Rodríguez-Vásquez y Font (2020) investigaron un profesor de matemáticas cuando enseña la derivada y temas relacionados con el Cálculo Diferencial. Precisamente este este trabajo es donde se encontró la nueva conexión metafórica con la cual se amplió la teoría de las conexiones matemáticas.

Particularmente, en los estudios relacionados con las conexiones sobre la derivada se identificó que los estudiantes de bachillerato, universitarios, futuros profesores y algunos profesores presentan dificultades para conectar significados parciales de la noción de derivada (FÉLIX-SANDOVAL y GRIJALVA-MONTEVERDE, 2021; PINO-FAN, GODINO y FONT, 2015; 2018; RODRÍGUEZ-NIETO et al., 2021; SÁNCHEZ-MATAMOROS, GARCÍA y LLINARES, 2008) y para conectar múltiples representaciones de la derivada, dado que no relacionan los registros gráficos con los analíticos (KULA-ÜNVER, 2020; PINO-FAN et al., 2015; 2018; PINO-FAN et al., 2017). Ahora bien, sobre la gráfica de la derivada, Berry y Nyman (2003) y Ubuz (2007) reportaron que los estudiantes tienen dificultades para conectar la representación gráfica de la función y la de su derivada. De hecho, Ubuz (2007), consideró que era difícil para los estudiantes utilizar la información gráfica para dar sentido a las representaciones simbólicas. Además, los estudiantes no comprenden la derivada porque tienen dificultades para relacionar tanto el crecimiento como el decrecimiento de $f$ y el signo de $f^{\prime}$ y $f^{\prime \prime}$ y trabajan más con información gráfica y el significado analítico puntual de la derivada (FUENTEALBA et al., 2018; FUENTEALBA, BADILLO y SÁNCHEZ-MATAMOROS, 2018).

Por su parte, Nemirovsky y Rubin (1992) reconocieron que muchos estudiantes dibujan la gráfica de $f^{\prime}$ de manera similar a la gráfica de $f$. Asimismo, Nemirovsky y Rubin (1992) y Oehrtman, Carlson y Thompson (2009) coinciden en que los estudiantes no consideran la relación lógica entre la función y su derivada y viceversa en un contexto gráfico. Ferrini-Mundy y Graham 
(1994) sostienen que los estudiantes desean hallar la ecuación de una función dada solo gráficamente, y, similarmente, lo reportaron García-García y Dolores-Flores (2019) quienes evidenciaron que los estudiantes del preuniversitario necesitan la expresión algebraica de la función para hacer una representación gráfica de ella misma y de su derivada, de lo contrario no grafican. En este sentido, se considera que es un aspecto clave investigar sobre las conexiones matemáticas teniendo en cuenta las propiedades de los gráficos, en especial cómo transitar de la representación gráfica de una función $g$ al gráfico de la derivada $g$ ' (GARCÍA-GARCÍA y DOLORES-FLORES, 2020).

La literatura muestra que la derivada es importante para el desarrollo del Cálculo (DOS SANTOS, 2020; VERÓN y GIACOMONE, 2021) y que los estudiantes presentan dificultades para comprenderla (PINO-FAN et al., 2015; RODRÍGUEZ-NIETO et al., 2021). De la revisión de la literatura, se concluye que los estudiantes proceden inadecuadamente cuando no se les da la expresión algebraica de la derivada o de la función (FERRINI-MUNDY y GRAHAM, 1994; VOSKOGLOU, 2017), pero sí muestran cierta comprensión cuando se les da la expresión algebraica. En esta línea, en esta investigación pretendemos respondemos a la pregunta ¿Dada la expresión algebraica de $g$, qué conexiones aseguran al estudiante una comprensión de la derivada que le permite realizar un esbozo de la gráfica de $g$ y explicar la relación que tiene la gráfica de $g$ con la gráfica de $g^{\prime}$ ?

\section{Marco teórico}

Esta investigación se fundamenta teóricamente en la conceptualización de comprensión matemática desde la articulación entre la Teoría Ampliada de las Conexiones (TAC) y el Enfoque Ontosemiótico de la Cognición e Instrucción matemática (EOS) (RODRÍGUEZ-NIETO et al., 2021).

\subsection{La comprensión en Educación Matemática}

La comprensión para Brownell y Sims (1946) es un término problemático dado que, "es muy difícil de encontrar o formular una definición técnicamente exacta de "comprender" 0 "comprensión" (p. 163). No obstante, a pesar del nivel de vaguedad que tiene esta noción, en el área de la Educación Matemática, la comprensión se ha caracterizado desde diferentes marcos 0 modelos teóricos. Por ejemplo, Polya (1962) propuso cuatro niveles de comprensión relacionados con la regla matemática: 1) nivel mecánico referido a la memorización y aplicación de un método de manera correcta, 2) nivel inductivo caracterizado por aceptar la indagación o exploración de casos simples o particulares se extienden a casos complejos, 3) nivel racional consiste en aceptar 
la prueba de la regla matemática y, en el 4) nivel intuitivo se refiere a "la convicción personal más allá de cualquier duda" (MEEL, 2003, p. 226).

Para Greeno (1978), la comprensión es un proceso caracterizado por sugerir "una estructura que representa las relaciones entre los componentes de la idea que se entiende" (p. 267). Además de este tipo de comprensión, que podemos llamar estructural, varios autores sugieren considerar, además, otro tipo de comprensión de tipo procedimental. En esta línea, tal como se señala en Borji, Radmehr y Font (2021) la literatura propone diversas formas de comprensión (conocer matemáticas y hacer matemáticas) que, a menudo, se definen con lenguaje dicotómico: instrumental versus relacional (SKEMP, 1976), operacional versus estructural (SFARD, 1991) y procedimental versus conceptual (HIEBERT y LEFEVRE, 1986). Por otra parte, en general, la comprensión relacional (estructural o conceptual) se considera como más deseable y valiosa (e.g., PESEK y KIRSCHNER, 2000).

Para Skemp (1976; 1978) existe una comprensión instrumental cuando el sujeto sabe hacer algo sin tener justificaciones o razones. En cambio, la comprensión relacional, se da cuando el sujeto sabe hacer algo y argumenta por qué debe hacerlo. Además, considera la comprensión lógica referida a la prueba formal en matemáticas y, la comprensión simbólica que alude a las conexiones de simbolismo y las notaciones relacionadas con las nociones matemáticas. En síntesis, para Skemp (1980) "comprender algo significa asimilarlo dentro de un esquema adecuado" (p. 50). Por su parte, Nickerson (1985) caracterizó la comprensión como la capacidad de visualizar propiedades de los conceptos, representar situaciones y usar modelos mentales; mientras que Michener (1978) asume que la comprensión es complementaria de la resolución de problemas matemáticos y enriquece los conocimientos considerando la creación de conexiones.

Polya (1989) definió la comprensión como un indicador que favorece a la resolución de problemas matemáticos, que de hecho, es el punto de partida en el método de cuatro fases sugerido para resolver problemas matemáticos: 1) comprender el problema para saber lo que se pide e identificar los datos, 2) identificar las relaciones entre los elementos del problemas como los datos y la incógnita de manera semántica y sintáctica como idea de resolución y trazar un plan, 3) ejecutar el plan propuesto y, 4) considerar una visión retrospectiva para revisar la solución del problemas y discutirla. Desde la perspectiva de Sierpinska (1990) la comprensión:

Se logra lentamente, junto con la acumulación de propiedades de los objetos, ejemplos y desarrollo de conceptos relacionados con las relaciones entre clases de conceptos. Al princípio, los conceptos en la memoria generalmente se definen solo parcialmente y se relacionan débilmente con otra información almacenada (p. 25).

Sierpinska (1990), define a la comprensión como un acto relacionado con procesos de interpretación entendidos como la dialéctica del desarrollo basada en la elaboración de 
suposiciones y su validación, y, estableció cuatro categorias fundamentadas en los actos de comprensión de un sujeto

IDENTIFICACIÓN de objetos que pertenecen a la denotación del (o: a) concepto (relacionado con el concepto en cuestión), o: identificación de un término con estatus científico; este acto consiste en una percepción repentina de algo que es como la "figura" en los experimentos Gestaltistas. DISCRIMINACIÓN entre dos objetos, propiedades, ideas, que antes se confundían. La GENERALIZACIÓN consiste en tomar conciencia de la no esencialidad de algún supuesto, o de la posibilidad de ampliar el abanico de aplicaciones. La SÍNTESIS consiste en captar las relaciones entre dos o más propiedades, hechos, objetos y organizarlos en un todo coerente (p. 29).

Posteriormente, Hiebert y Carpenter (1992) afirman que, la comprensión matemática se entiende como la red interna de representaciones de ideas o nociones matemáticas, procedimientos y hechos matemáticos. Esta definición de comprensión, está ligada a las conexiones entre representaciones internas y externas. 1) las conexiones entre representaciones externas se establecen teniendo en cuenta la similitud o diferencia "entre diferentes formas de representación de la misma idea matemática o entre ideas relacionadas dentro de la misma forma de representación" (HIEBERT y CARPENTER, 1992, p. 66). Mientras que, 2) las conexiones entre representaciones internas se evidencian cuando se construyen relaciones entre representaciones internas de ideas y procesan redes de conocimiento, relacionándose de manera jerárquica, relacionando casos particulares y generales (HIEBERT y CARPENTER, 1992). Pirie y Kieren (1989) desarrollaron un modelo donde definen la comprensión matemática de manera dinámica

Como nivelada (estable) pero no lineal. Es un fenómeno recursivo, y la recursión se observa cuando el pensamiento se mueve entre niveles de sofisticación. De hecho, cada nivel de comprensión se encuentra contenido dentro de los niveles subsiguientes. Cualquier nivel particular depende de las formas y procesos del mismo y, además, se encuentra restringido por los que están fuera de él (p. 8).

Además, Pirie y Kieren (1989; 1994) manifiestan que existen ocho niveles de comprensión matemática donde se puede avanzar y retroceder, los cuales son: 1) conocimiento primitivo, 2) creación de imágenes, 3) comprensión de la imagen, 4) observación de la propiedad, 5) formalización, 6) observación (el sujeto puede establecer conexiones entre conceptos matemáticos y reflexionar formalmente), 7) estructuración y, 8) invención.

Por otra parte, EI NCTM (2000) afirma que la comprensión de las matemáticas le permite al estudiante saber usar un objeto matemático en diferentes contextos de manera adecuada, organizar su conocimiento, hacer argumentaciones y establecer conexiones entre conceptos y procedimientos en la resolución de problemas intra y extramatemáticos. De esta manera, las conexiones matemáticas aparecen en la literatura como un indicador importante para la comprensión de conceptos matemáticos (BERRY y NYMAN, 2003; GARCÍA-GARCÍA y DOLORES-FLORES, 2019), en particular para el tipo de comprensión que se considera más 
valiosa o deseable (relacional, estructural, conceptual). El National Research Council [NRC] (2001) plantea que un estudiante con comprensión conceptual es consciente de la importancia de una idea matemática y la usa en un contexto apropiado. Asimismo, el estudiante sabe más que hechos aislados, es decir, organiza su conocimiento de forma coherente, permitiéndole aprender ideas nuevas y establecer conexiones matemáticas con ideas previas.

Para Kastberg (2002) la comprensión de un estudiante se relaciona con su colección de creencias sobre las matemáticas o bien, sobre un concepto matemático. Para este autor, la comprensión es evidenciada cuando los estudiantes usan representaciones y, propone cuatro categorías de evidencia de la comprensión: 1) concepción, 2) representación, 3) conexión y 4) aplicación. Particularmente, la conexión refiere a que un sujeto conecta dos representaciones cuando traduce una representación de un registro gráfico a otro simbólico o transforma una representación gráfica a otra en el mismo registro (KASTBERG, 2002). Con base en las ideas de Hiebert y Carpenter (1992), Nickerson (1985), entre otros autores, Meel (2003) afirma que

\footnotetext{
La formación de una red de conexiones proporciona una estructura para situar una nueva información mediante el conocimiento de similitudes, diferencias, relaciones inclusivas y relaciones de transferencia entre modelos. Por lo tanto, el desarrollo de la comprensión resulta un proceso de conectar las representaciones a una red cohesiva. El proceso de conexión requiere el conocimiento y los elementos de la red, así como de la estructura como un todo (p. 227).
}

Hiebert y Carpenter (1992) argumentan que, el nivel de comprensión matemática viene dado por el número y la fuerza de las conexiones. De hecho, la comprensión es un resultado del acto de hacer conexiones (CAI y DING, 2015). Por su parte, Chigeza (2013) afirma que "los estudiantes desarrollan la comprensión de las matemáticas cuando traducen entre y dentro de diferentes representaciones matemáticas" (p. 178). Otros marcos teóricos asumen la comprensión desde una vision constructivista y piagetiana sobre la abstracción reflexiva, por ejemplo, desde la teoría APOE, Arnon et al. (2014) sostienen que, para que un estudiante comprenda un determinado concepto matemático, debe tener la habilidad de transitar por construcciones mentales como la acción, proceso, objeto y esquema a través de seis mecanismos (interiorización, encapsulación, desencapsulación, reversión, coordinación y tematización), ver Figura 1.

Figura 1: Construcciones mentales y mecanismos en la teoría APOE

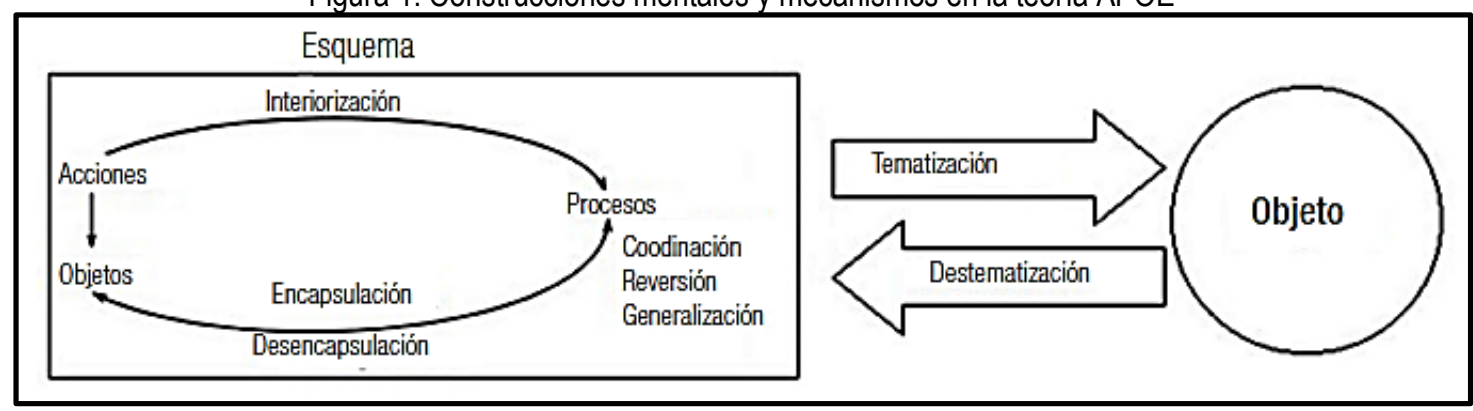

Fuente: Elaborado por Fuentealba et al. (2018, p. 6) basado en Arnon et al. (2014, p. 18). 
Generalmente, en la teoría APOE el proceso de comprensión matemática inicia cuando el sujeto manipula objetos físicos o mentales que hacen referencia a las acciones (A). Luego, estas acciones el sujeto las interioriza en procesos $(P)$ que serán encapsulados en objetos $(0)$. Posteriormente, las acciones, los procesos y los objetos construidos por el sujeto, se organizan en esquemas (E) (ARNON et al., 2014). Aunado a este proceso de comprensión matemática desde la teoría APOE, se consideran dos aspectos importantes como la descomposición genética que es un modelo que permite la descripción y predicción para construir un concepto matemático; la noción de esquema como estructura dinámica en constante cambio, evolución y su desarrollo depende de los niveles: intra, inter y trans (ARNON et al., 2014).

Por otra parte, en los acuerdos cognitivos sobre el aprendizaje de las matemáticas, el Instituto Nacional de Formación Docente [INFD] (2010) planteó que

Comprender un objeto matemático significa haber transitado por diversas experiencias
que le permitan al estudiante producir, organizar y re-organizar la red de relaciones
que se deben establecer en la resolución de una situación problemática que "obliga"
al funcionamiento del objeto, los procedimientos o técnicas que se despliegan para
resolverla, las definiciones, propiedades, argumentos que validan las acciones
realizadas, todas ellas soportadas y reguladas por el lenguaje simbólico, propio de la
Matemática, y la lengua natural (p. 122).

En la revisión de la literatura, tal como se afirma en Font (2007), básicamente hay dos maneras de entender la "comprensión": como proceso mental o como competencia. Desde la TAC, se tienen en cuenta estas dos perspectivas y se considera que la comprensión de un concepto matemático se logra cuando el sujeto hace conexiones matemáticas para resolver problemas (GARCÍA-GARCÍA y DOLORES-FLORES, 2019; RODRÍGUEZ-NIETO et al., 2021).

A su vez, en el EOS, en cierta manera, también se consideran estas dos perspectivas. Los posicionamientos pragmatistas del EOS llevan a entender, de entrada, la comprensión básicamente como competencia y no tanto como proceso mental. Se considera que un sujeto comprende un determinado objeto matemático, cuando lo usa de manera competente en diferentes prácticas, lo cual implica concebirla también como "conocimiento y aplicación de las normas" que regulan la práctica. Por otra parte, dado que en estas prácticas se activan objetos primarios (problemas, notaciones, proposiciones, definiciones, procedimientos y argumentos) que se relacionan por medio de funciones semióticas, también se tiene en cuenta el papel esencial que las funciones semióticas tienen en el proceso relacional entre los objetos matemáticos activados en las prácticas, $y$, por tanto, también se interpreta la comprensión de un objeto matemático 0 por parte de un sujeto $X$ (persona 0 institución) en términos de las funciones semióticas que $X$ puede establecer, en unas circunstancias fijadas, en las que se pone en juego 0 como funtivo (expresión o contenido) (ORTIZ y FONT, 2011). 
Integrando las posturas sobre comprensión de la TAC (RODRÍGUEZ-NIETO et al., 2021) y el EOS (FONT, 2007; ORTIZ y FONT, 2011; GODINO, BATANERO y FONT, 2019), consideramos que la comprensión de una noción matemática se evidencia (en) y emerge (de) la actividad matemática en la que un sujeto relaciona objetos primarios (situaciones problemas, conceptos/definiciones, proposiciones/propiedades, procedimientos, representaciones, argumentos) por medio de funciones semióticas. Dicha comprensión le permite al sujeto usar esta noción matemática de manera competente en la resolución de problemas. Por otra parte, las prácticas matemáticas, los procesos que realiza, los objetos primarios que activa en estas prácticas y las funciones semióticas que los relacionan se pueden agrupar en algunos de los tipos de conexiones que considera la TAC (ver sección 2.2). De esta manera, se puede afirmar que la realización de conexiones asegura la comprensión del sujeto y que el no establecimiento de determinadas conexiones es una explicación plausible de la falta de comprensión en el sujeto.

\subsection{Teoría Ampliada de las Conexiones Matemáticas (TAC)}

Inicialmente la TAC toma como definición de conexión matemática la postura de Businskas (2008) quien asume que una conexión matemática es entendida como "una relación verdadera entre dos ideas matemáticas, $A$ y $B$ " (p. 18). Posteriormente, esta definición fue ampliada por García-García y Dolores-Flores (2018) afirmando que, una conexión matemática es "un proceso cognitivo a través del cual una persona relaciona dos o más ideas, conceptos, definiciones, teoremas, procedimientos, representaciones y significados entre sí, con otras disciplinas o con la vida real" (GARCÍA-GARCÍA y DOLORES-FLORES, 2018, p. 229). Luego, Kenedi et al. (2019) caracterizaron el término conexiones como aquellas que forman parte de una red de información que se interrelaciona con otras ciencias, útiles para comprender y establecer relaciones entre ideas, conceptos y procedimientos matemáticos. En esta investigación consideramos a la conexión matemática desde una perspectiva integradora desde la TAC y el EOS, tal como se hace en RODRÍGUEZ-NIETO et al. (2021). Estos autores definieron metafóricamente a las conexiones matemáticas como la punta de un iceberg conformadas por un conglomerado de prácticas, procesos, objetos primarios identificados en la actividad matemática de un sujeto cuando resuelve una tarea (intra o extramatemática) y funciones semióticas que los relacionan.

En la TAC se consideran diez tipos de conexiones matemáticas que han emergido de los resultados de diferentes investigaciones sobre esta temática (BUSINSKAS, 2008; ELI et al., 2013; EVITTS, 2004; GARCÍA-GARCÍA y DOLORES-FLORES, 2019; 2020; RODRÍGUEZ-NIETO et al., 2020; 2021), las cuales se presentan en la Tabla 1 (Orientada a la instrucción (OI), Modelado (MD), 
Procedimental (P), Representaciones diferentes (RD), Parte-todo (P-T), Implicación (I), Característica (C), Reversibilidad (R), Significado (S) y Metafórica (MT)). Cabe destacar que, estas conexiones, pueden ser intramatemáticas que "se establecen entre ideas, conceptos, procedimientos, teoremas, representaciones y significados matemáticos entre sí" (DOLORESFLORES y GARCÍA-GARCÍA, 2017, p. 160), y, extramatemáticas identificadas cuando "se relaciona un concepto o modelo matemático con un problema en contexto o de aplicación 0 , viceversa. Incluyen las conexiones entre contenidos matemáticos con otras disciplinas curriculares y con situaciones de la vida diaria" (DOLORES-FLORES y GARCÍA-GARCÍA, 2017, p. 161).

Tabla 1: Conexiones matemáticas (CM) propuestas en la TAC

\begin{tabular}{|c|c|}
\hline CM & Descripción \\
\hline OI & $\begin{array}{l}\text { Se refiere a la comprensión de un concepto } C \text { con base en dos o más conceptos previos } A \text { y } B \text {, } \\
\text { requeridos para ser entendidos por un estudiante. Además, estas conexiones se pueden } \\
\text { identificar de dos formas: 1) la asociación de un nuevo tema con conocimientos previos, y, 2) los } \\
\text { conceptos y procedimientos matemáticos conectados entre sí se consideran prerrequisitos o } \\
\text { habilidades que los estudiantes deben dominar antes del desarrollo de un nuevo concepto } \\
\text { (BUSINSKAS, 2008). }\end{array}$ \\
\hline MD & $\begin{array}{l}\text { Son relaciones entre las matemáticas y la vida real y se evidencian cuando el sujeto resuelve } \\
\text { problemas no matemáticos o de aplicación donde tiene que plantear un modelo o expresión } \\
\text { matemática (EVITTS, 2004). }\end{array}$ \\
\hline$P$ & $\begin{array}{l}\text { Se manifiestan mediante el uso de reglas, algoritmos o fórmulas para resolver una tarea } \\
\text { matemática. Estas conexiones son de la forma, } A \text { es un procedimiento utilizado para trabajar } \\
\text { con el concepto } B \text { (BUSINSKAS, 2008; GARCÍA-GARCÍA y DOLORES-FLORES, 2019). Por } \\
\text { ejemplo, la derivada de } g(x)=a^{x} \text { se encuentra a través de la fórmula } g^{\prime}(x)=a^{x} \ln (a) \text {. }\end{array}$ \\
\hline & $\begin{array}{l}\text { Son identificadas cuando el sujeto representa los objetos matemáticos usando representaciones } \\
\text { equivalentes y alternas (BUSINSKAS, 2008). 1) Las representaciones equivalentes son } \\
\text { transformaciones de representaciones en un mismo registro (e.g., } Q(x)=x^{2}+6 x+9 \text { es } \\
\left.\text { equivalente a } Q(x)=(x-3)^{2}\right) \text {. 2) Las representaciones alternas se refieren a } \\
\text { representaciones de un mismo objeto donde se modifica el registro en el cual fueron formadas } \\
\text { inicialmente (e.g., gráfica-algebraica) (BUSINSKAS, 2008), por ejemplo, la representación } \\
\text { gráfica de } f(x)=\operatorname{sen}(x)+e^{x} \text {, ver Figura 2. }\end{array}$ \\
\hline
\end{tabular}

Figura 2. Representaciones alternas.

$\mathrm{RD}$

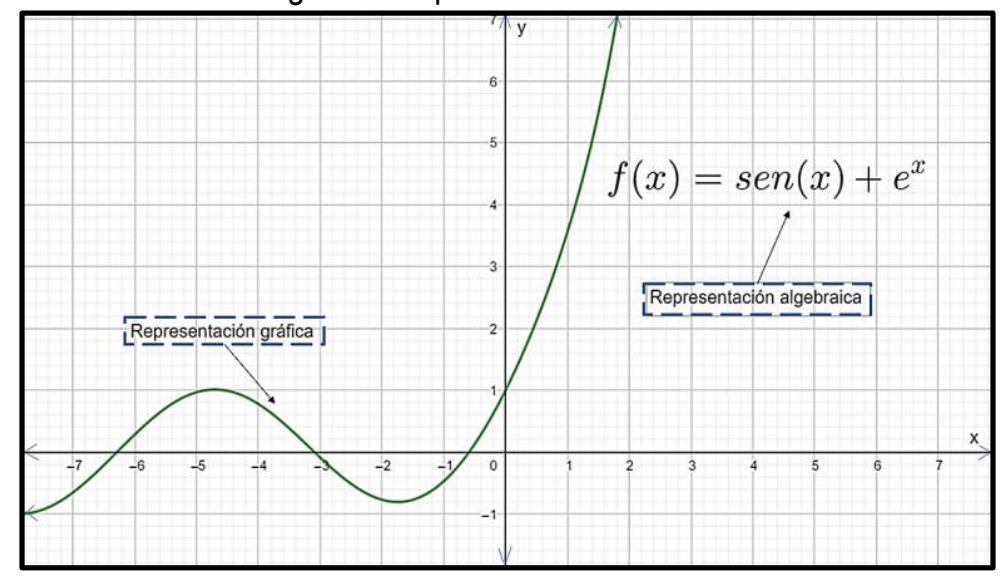

Fuente: Elaboración propia usando GeoGebra. 


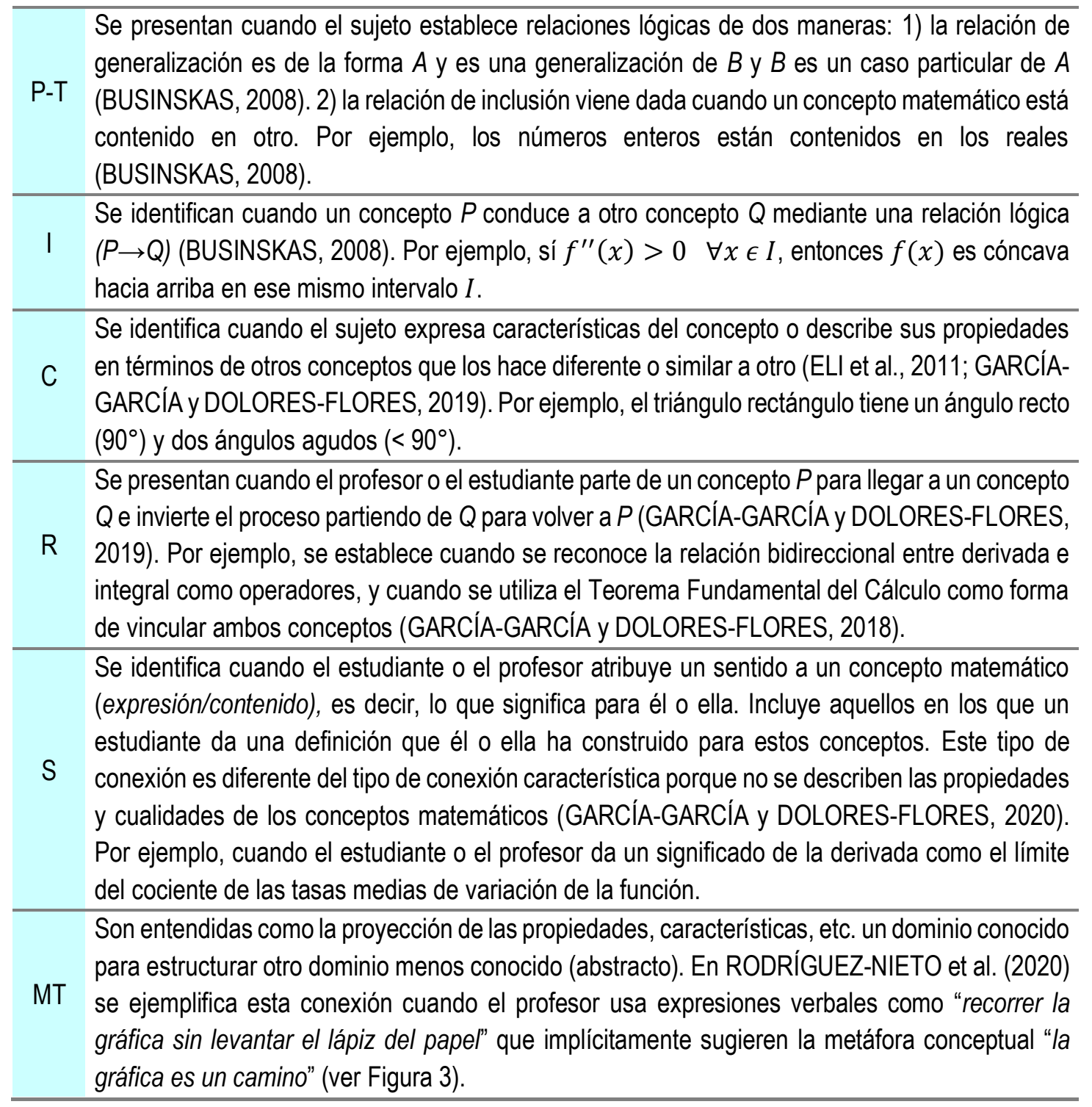

Fuente: Tomado de Businskas (2008), García-García y Dolores-Flores (2019) y Rodríguez-Nieto et al. (2021).

Las conexiones matemáticas se pueden identificar en las producciones escritas de los sujetos cuando resuelven una tarea matemática, así como en sus argumentos verbales y gestuales (GARCÍA-GARCÍA y DOLORES-FLORES, 2018). Es oportuno mencionar que "la investigación en Educación Matemática puede validar estas tipologías, pero, también, podría incluir otras categorías aun no identificadas" (GARCÍA-GARCÍA, 2019, p. 131). 
Figura 3: Caracterización y proyección de la conexión metafórica.

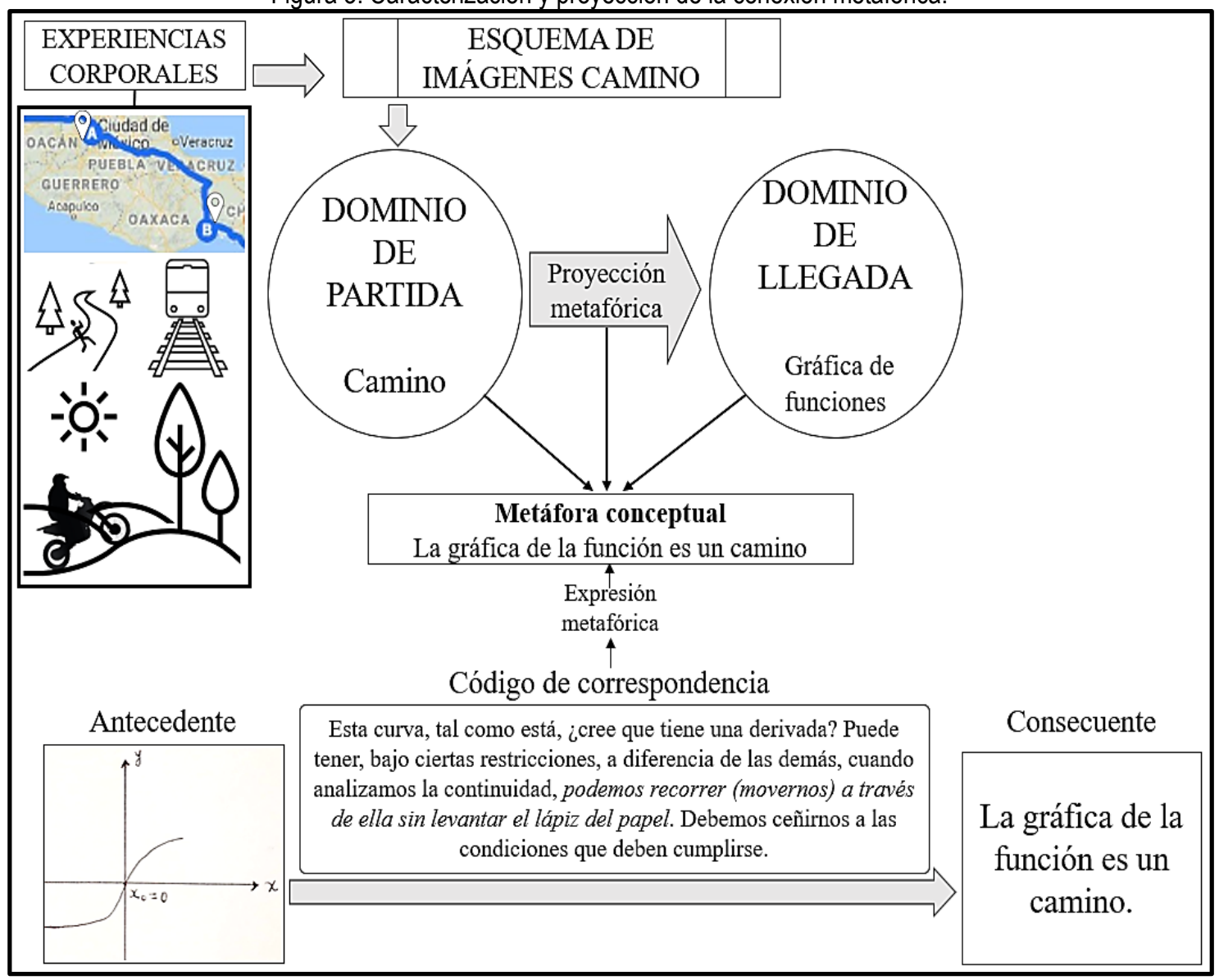

Fuente: Tomado de Rodríguez-Nieto et al. (2020).

\subsection{Algunas herramientas del Enfoque Ontosemiótico (EOS)}

EI EOS es un sistema teórico inclusivo y articulador sobre el conocimiento y la instrucción matemática, el cual fue impulsado por la necesidad de clarificar y mejorar nociones teóricas y metodológicas de diferentes marcos teóricos usados en Educación Matemática desde uma perspectiva unificada (GODINO y BATANERO, 1994). También, este enfoque surge para responder a preguntas: ¿Qué es un objeto matemático? ¿Cuál es el significado de un objeto matemático?; o bien, ¿Qué tipos de objetos intervienen en la actividad matemática de un sujeto? (GODINO et al., 2021; GODINO et al., 2019).

En el EOS, según Font, Godino y Gallardo (2013), la actividad matemática se puede entender desde una perspectiva institucional o personal, modelada en términos de prácticas y de configuración de objetos primarios y procesos activados en dichas prácticas. La práctica matemática es "toda situación o expresión (verbal, gráfica, simbólica) realizada por alguien para resolver problemas matemáticos, comunicar a otros la solución obtenida, validarla o generalizarla a otros contextos y problemas" (GODINO y BATANERO, 1994, p. 334). En estas prácticas intervienen objetos primarios - entendidos en un sentido amplio como cualquier entidad 
involucrada en la actividad matemática y que puede reconocerse como una unidad (FONT et al., 2013), por ejemplo: problemas, notaciones, definiciones, proposiciones procedimientos y argumentos. Los objetos primarios que emergen en la práctica matemática pueden hacerlo de diferentes maneras, las cuales son el resultado de las distintas maneras de ver, hablar, operar, etc. sobre los objetos primarios; lo cual permite hablar de objetos primarios personales 0 institucionales, ostensivos o no ostensivos, unitarios o sistémicos, intensivos o extensivos y de contenido o expresión (GODINO, BATANERO y FONT, 2007). En el EOS se tienen en cuenta seis objetos primarios (ver Tabla 2), que relacionados entre sí forman configuraciones de objetos primarios (GODINO et al., 2019).

Tabla 2: Objetos primarios desde la perspectiva del EOS

\begin{tabular}{cl}
\hline \multicolumn{1}{c}{ Objetos primarios } & \multicolumn{1}{c}{ Descripción } \\
\hline $\begin{array}{c}\text { Situaciones problemas/Tarea } \\
(\mathrm{T})\end{array}$ & Tareas intra y extramatemáticas, ejercicios, ejemplos, preguntas, etc. \\
\hline Elementos lingüísticos (E-L) & $\begin{array}{l}\text { Términos, expresiones, notaciones, gráficos, etc. en sus distintos } \\
\text { registros (escrito, oral o verbal, gestual, gráfico, tabular, simbólico, etc.) }\end{array}$ \\
\hline $\begin{array}{c}\text { Conceptos/Definiciones (D) } \\
\text { Proposiciones/propiedades } \\
(\mathrm{Pr})\end{array}$ & $\begin{array}{l}\text { Son introducidos mediante definiciones o descripciones, explícitas o no } \\
\text { (recta, punto, función, derivada, pendiente, función, etc.) }\end{array}$ \\
\hline Procedimientos (Pc) & Algoritmos, operaciones, técnicas de cálculo, usar fórmulas, reglas, etc. \\
\hline Argumentos (A) & $\begin{array}{l}\text { Son enunciados utilizados para validar o explicar las proposiciones y } \\
\text { procedimientos, ya sean deductivos o de otro tipo. }\end{array}$ \\
\hline
\end{tabular}

Una configuración está definida como "las redes de objetos intervinientes y emergentes de los sistemas de prácticas. Estas configuraciones pueden ser epistémicas (redes de objetos institucionales) o cognitivas (redes de objetos personales)" (GODINO et al., 2010, p. 254). La configuración epistémica es el sistema de objetos primarios que, desde una perspectiva institucional están involucrados en las prácticas matemáticas llevadas a cabo para resolver un problema específico y la configuración cognitiva es el sistema de objetos matemáticos primarios que un sujeto moviliza como parte de las prácticas matemáticas desarrollas para resolver un problema específico (GODINO et al., 2019).

El conjunto de objetos primarios de la Tabla 2 emerge en la actividad matemática a través de la activación de procesos matemáticos primarios (comunicación, planteamiento de problemas, definición, enunciación, elaboración de procedimientos y argumentación) derivados de la aplicación de la perspectiva proceso-producto a dichos objetos (GODINO et al., 2007). Estos procesos se dan juntamente con los derivados de aplicar la dualidad proceso-producto a las cinco dualidades comentadas anteriormente (institucional/personal, expresión/contenido, ostensivo/no ostensivo, unitario/sistémico y extensivo/intensivo): personalización-institucionalización; síntesis- 
análisis; representación-significado; materialización-idealización; generalización-particularización (FONT et al., 2013; FONT et al., 2016; GODINO et al., 2007; GODINO et al., 2010; LUGOARMENTA, PINO-FAN y RUIZ, 2021), ver Figura 4. Para Godino et al. (2007), la resolución de problemas y el modelado matemático deben denominarse "hiperprocesos" matemáticos que combinan algunos de los procesos comentados.

Figura 4: Representación ontosemiótica del conocimiento matemático Institucional

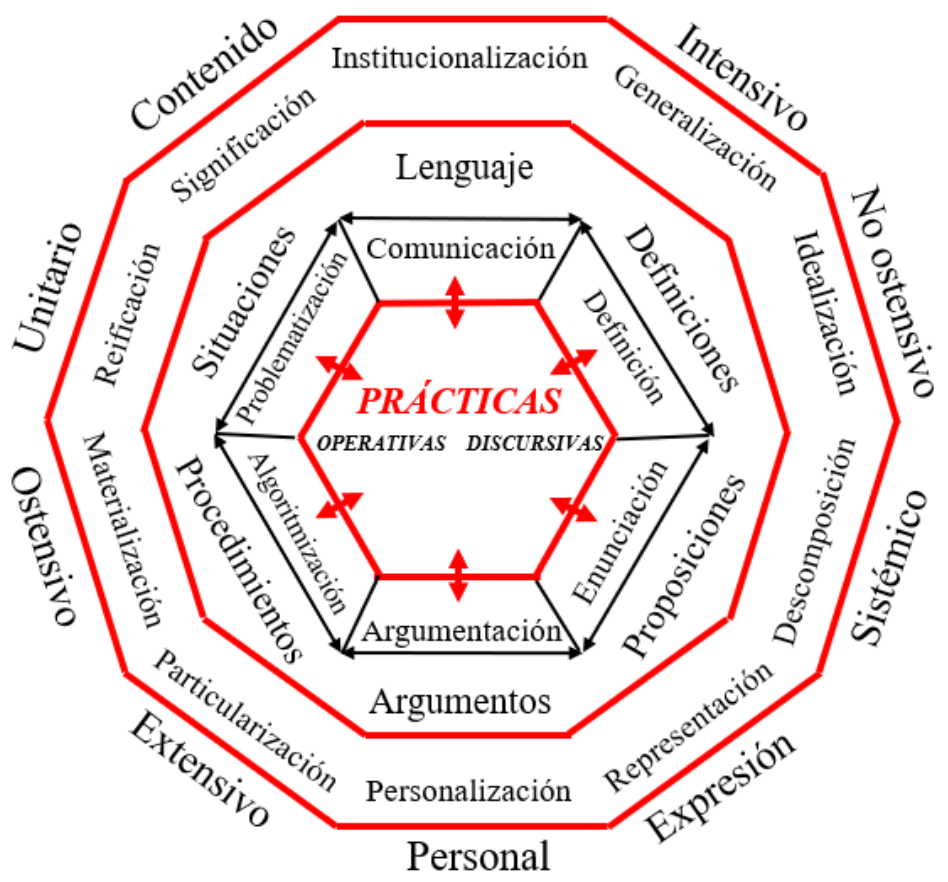

Fuente: Tomado de Font y Contreras (2008)

En el EOS se considera fundamental la noción de función semiótica que permite relacionar las prácticas con los objetos y procesos que se activan y admite construir una noción operativa del conocimiento, significado, comprensión y competencia (GODINO et al., 2007). Una función semiótica es una relación triádica entre un antecedente (expresión/objeto inicial) y un consecuente (contenido/objeto final) establecida por un sujeto (persona o institución) de acuerdo con un cierto criterio o código de correspondencia (FONT, 2007). Por ejemplo, dado un objeto matemático (expresión), el significado es el objeto matemático considerado como contenido (GODINO et al., 2010). Las funciones semióticas se infieren cuando se observa la actividad matemática desde la dualidad expresión/contenido (ver esquema en la Figura 5).

Figura 5: Esquema de la función semiótica

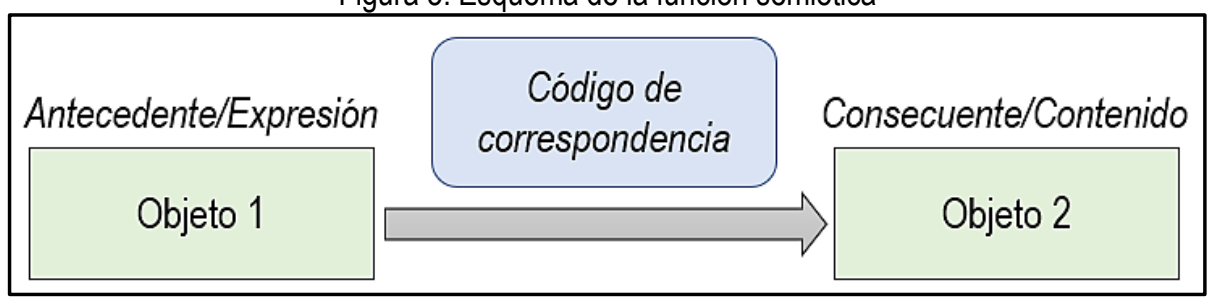

Fuente: Tomado de Rodríguez-Nieto et al. (2020) 
En el networking entre la TAC y el EOS (RODRÍGUEZ-NIETO et al., 2021), la función semiótica es más general que la noción de conexión matemática, dado que las conexiones se consideran casos particulares de funciones semióticas de carácter personal o institucional. En la TAC, la conexión matemática puede ser verdadera o no, lo cual se reinterpreta desde el EOS de la siguiente manera: cuando un sujeto hace una conexión correcta coincide con la institucional y cuando es incorrecta solo es de tipo personal.

\section{Metodología}

Esta investigación es de tipo cualitativa (COHEN, MANION y MORRISON, 2018), enfocada en analizar la comprensión de estudiantes universitarios cuando resuelven una tarea sobre la derivada dada la expresión algebraica de la función $g$. El estudio se desarrolló en tres etapas: 1) selección de los participantes, 2) diseño de la tarea en la que debían esbozar la gráfica de $g$ y explicar la relación que tiene con la de la derivada y aplicación de las entrevistas semiestructuradas, y, 3) análisis de la actividad matemática de los estudiantes para identificar las conexiones y su comprensión matemática.

\subsection{Participantes y contexto}

Los participantes fueron dos estudiantes (una mujer y un hombre) con edad promedio de 19 años, quienes cursaban el primer año de Licenciatura en Matemáticas de una Universidad del suroeste mexicano. Fueron seleccionados porque en su primer semestre de la carrera, habían aprobado la asignatura de Cálculo Diferencial donde se desarrolló el tema de la derivada y sus aplicaciones. Además, la coordinación de la Facultad de Matemáticas permitió que el primer autor de este artículo ingresara al aula de clases e interactuar con los estudiantes.

\subsection{Método de recolección de datos}

Para la recolectar la información primero se diseñó una tarea sobre la expresión algebraica de la función para hallar la derivada y las gráficas de $g$ y $g^{\prime}$ y, luego, se aplicaron entrevistas semiestructuradas cuando los estudiantes resolvian la tarea propuesta. Se optó por las entrevistas semiestructuradas porque permiten establecer un dialogó entre el investigador y los participantes con el fin de profundizar sobre el tema de interés (COHEN et al., 2018). Cabe destacar que, el investigador (I) hacia preguntas con base en las respuestas de los entrevistados (E1 y E2). 


\subsubsection{Tarea}

La tarea se propuso para explorar las conexiones matemáticas y la comprensión cuando los estudiantes la resolvieran. Además, se consideró lo sugerido por la literatura sobre las conexiones y la derivada, esto es, que la mayoría de los estudiantes no hacen la gráfica de $g \circ g^{\prime}$ porque no se les suministra la expresión algebraica.

Tarea: Dada la función $g(x)=\frac{x^{3}}{3}-2 x^{2}+3 x+1$, a) encuentre los intervalos en los que $g$ es creciente o decreciente, b) determine dónde la función tiene máximo relativo o mínimo relativo, c) halle los puntos de inflexión de la función, d) determine dónde la gráfica es cóncava hacia arriba o cóncava hacia abajo, e) realice una gráfica de la función derivada $g^{\prime}, y, f$ ) explique ampliamente ¿Qué relación tiene la gráfica de $g$ con la gráfica de $g$ '?

\subsection{Método de análisis de datos}

El análisis de datos se llevó a cabo teniendo en cuenta dos métodos. Por una parte, se usó el análisis temático considerado por la TAC donde se siguen seis fases (familiarización con los datos, generación de códigos iniciales, búsqueda de temas, revisión de temas, definición y nombramiento de temas y el reporte) (BRAUN y CLARKE, 2006), y por otra, se utilizó el modelo de análisis de la actividad matemática propuesto por el EOS. Ambos métodos, según RodríguezNieto et al. (2021), básicamente, son análisis de contenido temático. En este sentido, en el análisis temático de la TAC, se utiliza una tipología de conexiones matemáticas establecidas a priori en el marco teórico, mientras que el análisis realizado con el EOS utiliza diversas herramientas (prácticas, objetos, procesos y funciones semióticas). Ahora bien, aunque el nivel de detalle de los dos métodos de análisis es diferente, ambos son complementarios. No obstante, en esta investigación sólo se considerarán: la primera fase del análisis temático (TAC) y los siguientes tipos de análisis de la actividad matemática de los estudiantes propuestos en el EOS: prácticas, configuraciones de objetos primarios y funciones semióticas (ver Tabla 3).

Tabla 3: Método para analizar la actividad matemática

\begin{tabular}{ccl}
\hline \multicolumn{2}{c}{ Fases } & \multicolumn{1}{c}{ Descripción } \\
\hline $\begin{array}{c}\text { Transcripción } \\
\text { de las } \\
\text { entrevistas }\end{array}$ & $\begin{array}{l}\text { Las entrevistas realizadas se transcriben y se leen para que el investigador se } \\
\text { familiarice con los datos recolectados. Además, se revisan las producciones escritas, } \\
\text { que, de hecho, es la primera fase del análisis temático de la TAC. }\end{array}$ \\
\hline 2 & $\begin{array}{l}\text { Narrativa } \\
\text { temporal }\end{array}$ & $\begin{array}{l}\text { Se explica matemáticamente, lo que hace el sujeto cuando resuelve la tarea. En ella } \\
\text { se encuentran las prácticas realizadas por el estudiante o el profesor y algunos objetos } \\
\text { primarios que, metafóricamente, juegan el papel de los protagonistas de la narrativa. }\end{array}$ \\
\hline 3 & $\begin{array}{l}\text { Prácticas } \\
\text { matemáticas }\end{array}$ & $\begin{array}{l}\text { A partir de la narrativa se describen las prácticas matemáticas entendidas como una } \\
\text { secuencia de acciones, reguladas por reglas institucionalmente establecidas, } \\
\text { orientadas hacia la resolución de un problema (FONT et al., 2013). }\end{array}$ \\
\hline
\end{tabular}


4

Configuración cognitiva

Se construye la configuración cognitiva que es el sistema de objetos matemáticos primarios que un sujeto moviliza como parte de las prácticas matemáticas desarrollas para resolver un problema específico (GODINO et al., 2019).

5

Funciones Se establecen las funciones semióticas entre los objetos primarios de la configuración semióticas cognitiva.

Fuente: Adaptado de Rodríguez-Nieto et al. (2021)

\section{Resultados}

Los resultados se presentarán siguiendo las fases de análisis de la Tabla 3. La primera fase del método no se muestra porque se usará para dar evidencias en el desarrollo de las otras fases (2 hasta la 5). Además, solo se mostrarán los resultados completos de E1 y, del E2 se reflexionará sobre su comprensión, prácticas y conexiones matemáticas.

\subsection{Narrativa}

Se le propuso la tarea al estudiante E1, quien la leyó y la comprendió, dado que inicialmente entendió la derivada como la pendiente de la recta tangente a la curva en un punto y el punto crítico donde la derivada se hace cero. Luego, calculó la primera derivada de la función $g(x)=\frac{x^{3}}{3}-2 x^{2}+3 x+1$, obteniendo como resultado $g^{\prime}(x)=x^{2}-4 x+3$. Este polinomio cuadrático, E1 lo igualó a cero y completando cuadrado encontró los puntos críticos $(x=1$ y $x=3)$. Posteriormente, determinó los intervalos de crecimiento $(-\infty, \mathbf{1})$ y $(3,+\infty)$ y decrecimiento $(\mathbf{1}, \mathbf{3})$ evaluando a $g$ en valores cercanos a los puntos críticos, y, con base en esta información, E1 halló el punto máximo de $g$ que se encuentra en $x=1$ y el punto mínimo en $x=$ 3. Seguidamente, calculó la segunda derivada y la igualó a cero $g^{\prime \prime}(x)=2 x-4=0$, con el fin

de encontrar el punto de inflexión $\left(2, \frac{5}{3}\right)$. En este contexto, E1 asumió que el punto de inflexión es aquel donde $g$ cambia de concavidad y, con esto, determinó los intervalos donde $g$ es cóncava hacia abajo $(-\infty, 2)$ y cóncava hacia arriba $(2,+\infty)$. Por último, con base en la información obtenida, E1 dibujó las gráficas de $g$ y $g^{\prime}$, y para ello, trazó un plano cartesiano, ubico puntos, elaboró una tabla de valores que le permitió encontrar otros puntos y dibujar las gráficas.

\subsection{Prácticas matemáticas $(\mathrm{Pm})$}

En la Tabla 4 se presentan las prácticas matemáticas realizadas por el estudiante E1 cuando resolvió la tarea propuesta.

Tabla 4: Descripción de las prácticas matemáticas de E1

\begin{tabular}{cl}
\hline Pm & \multicolumn{1}{c}{ Acciones secuenciadas } \\
\hline \multirow{2}{*}{ Pm1 } & $\begin{array}{l}\text { El estudiante E1 leyó y comprendió la tarea propuesta (Comprendió la derivada como la } \\
\text { pende de la recta tangente a una curva en un punto y el punto crítico como el valor en } x \\
\text { donde la derivada se hace cero). }\end{array}$ \\
\hline
\end{tabular}


Calculó la derivada de la función $g(x)=\frac{x^{3}}{3}-2 x^{2}+3 x+1$, obteniendo como resultado Pm2 $g^{\prime}(x)=x^{2}-4 x+3$. Para ello, implícitamente usó la fórmula $\frac{d\left(x^{n}\right)}{d x}=n x^{n-1}$ y reglas de derivación: derivada de la suma de dos funciones y derivada de una función multiplicada por una constante.

Calculó los puntos críticos de la función. Para ello, consideró el criterio de la primera derivada,

Pm3 igualó a cero la derivada $x^{2}-4 x+3=0$, completó cuadrado, realizó algunas operaciones algebraicas, aritméticas y expresiones equivalentes, hasta encontrar los puntos ( $x=1$ y $x=3$ ). Determinó los intervalos de crecimiento $(-\infty, \mathbf{1}) y(\mathbf{3},+\infty)$ y decrecimiento $(\mathbf{1}, \mathbf{3})$. Para lograrlo, consideró el criterio de la primera derivada y sustituyó $x=0$ en $g$ y obtuvo $g^{\prime}(0)=$ $3>0$, entonces afirmó que en $(-\infty, 1)$ la función es creciente. Luego, sustituyó $x=2$ en $g$ y obtuvo $g^{\prime}(2)=-1<0$, entonces afirmó que, en $(\mathbf{1}, \mathbf{3})$ la función es decreciente. Por último, sustituyó $x=4$ en $g$ y obtuvo $g^{\prime}(4)=3>0$, entonces afirmó que, en $(3,+\infty)$ la función es creciente.

Con base en el punto crítico $x=1$, E1 encontró el punto máximo. Para ello usó el criterio de la primera derivada y sustituyó $x=0$ en $g^{\prime}$, que es un valor antes de $x=1$, de lo cual obtuvo

Pm5 $g^{\prime}(0)=3>0$ (positivo). Luego, sustituyó $x=2$ en $g^{\prime}$ que es un valor después de $x=1$, obteniendo $g^{\prime}(2)=-1<0$ (negativo). Por lo tanto, E1 afirmó que en $x=1, g$ tiene un máximo.

Pm6 $\left(1, \frac{7}{3}\right)$.

Sustituyó $x=1$ en $g$, realizó operaciones aritméticas y encontró que el punto máximo es

Con en el punto crítico $x=3$, E1 encontró el punto mínimo. Sustituyó $x=4$ (valor después de

Pm7 $x=3$ ) en $g^{\prime}$, obteniendo $g^{\prime}(4)=3>0$ (positivo). Por lo tanto, E1 afirmó que en $x=3, g$ tiene un mínimo.

Pm8 Sustituyó $x=3$ en $g$, realizó operaciones aritméticas y halló el punto mínimo $(3,1)$.

Pm9 Encontró el punto de inflexión por medio del criterio de la segunda derivada. Es decir, halló la segunda derivada de $g$, la igualó a cero $g^{\prime \prime}(x)=2 x-4=0$, despejó y obtuvo que $x=2$.

Pm10

Evaluó $x=2$ en $g$ y obtuvo la imagen $\frac{5}{3}$, lo cual le permitió encontrar el punto de inflexión $\left(2, \frac{5}{3}\right)$.

Determinó los intervalos de concavidad $(-\infty, 2)$ y $(2,+\infty)$. Para ello, uso el criterio de la segunda derivada, mencionando que, si la segunda derivada en un intervalo es menor que cero entonces la función es cóncava hacia abajo, por ejemplo, E1 evaluó a $x=1$ en $g$ " que es un

Pm11 valor que está antes de $x=2$ y obtuvo que $g$ " $(1)=-2<0$. Por lo tanto, $g$ es cóncava hacia abajo en $(-\infty, 2)$. Posteriormente, evaluó $x=3$ en $g$ " que es un valor que está después de $x=2$, obteniendo que $g^{\prime \prime}(3)=2>0$. Por lo tanto, $g$ es cóncava hacia arriba en $(2,+\infty)$.

Pm12 Identificó que el punto en $x=2$ si es el punto de inflexión porque genera el cambio de ser cóncava hacia abajo a cóncava hacia arriba.

Pm13 Para hacer la gráfica de $g$, E1 primero dibujó un sistema de coordenadas cartesianas.

Pm14 Ubicó los intervalos donde $g$ es creciente y decreciente.

Pm15 Ubicó en el plano el punto máximo $\left(1, \frac{7}{3}\right)$ y punto mínimo $(3,1)$ y los puntos de corte con el eje $x$.

Pm16 Construyó una tabla de valores para graficar a $g(x)=\frac{x^{3}}{3}-2 x^{2}+3 x+1$. 
Pm17 Ubicó los puntos obtenidos en la tabla de valores en el plano cartesiano.

Pm18 Dibujó la gráfica de $g(x)=\frac{x^{3}}{3}-2 x^{2}+3 x+1$.

Pm19 Para hacer la gráfica de $g^{\prime}$, E1 hizo un sistema de coordenadas cartesianas.

Pm20 Ubico los puntos en el plano donde la derivada se hace cero: $(1,0)$ y $(3,0)$.

Pm21 Construyó una tabla de valores para graficar a $g^{\prime}(x)=x^{2}-4 x+3$.

Pm22 Ubicó los puntos obtenidos en la tabla de valores en el plano cartesiano.

Pm23 Dibujó la gráfica de $g^{\prime}(x)=x^{2}-4 x+3$.

Pm24 Enunció características de las funciones, afirmando que ambas funciones son continuas, que la gráfica de $g$ tiene un máximo y un mínimo, y la gráfica de $g$ ' solo tiene un mínimo.

Pm25 Enunció que la derivada es una función de grado menor que la función original.

Fuente: Elaboración propia

\subsection{Configuración cognitiva de E1}

Ahora bien, con base en la narrativa y descripción de las prácticas matemáticas, se construye la configuración cognitiva de E1 considerando los objetos primarios (OP) (ver Tabla 5).

Tabla 5: Descripción detallada de los objetos primarios

\begin{tabular}{ll}
\hline OP & \multicolumn{1}{c}{ Descripción } \\
\hline & Dada la función $g(x)=\frac{x^{3}}{3}-2 x^{2}+3 x+1$, \\
& T1: a) Encuentre los intervalos en los que $g$ es creciente o decreciente. \\
T2: b) Determine dónde la función tiene máximo relativo o mínimo relativo. & T3: c) Halle los puntos de inflexión de la función. \\
T4: d) Determine dónde la gráfica es cóncava hacia arriba o cóncava hacia abajo. \\
T5: e) Realice una gráfica de la función derivada $g^{\prime}$. \\
T6: f) Explique ampliamente ¿Qué relación tiene la gráfica de $g$ con la gráfica de $g$ '? \\
\hline
\end{tabular}

Verbal: punto, punto crítico, punto máximo, punto mínimo, punto de inflexión, recta, gráfica, ecuación, recta tangente, derivada, segunda derivada, derivada en un punto, concavidad hacia arriba y hacia abajo, función creciente y decreciente, intervalo, plano cartesiano...

Simbólico: $g(x)=\frac{x^{3}}{3}-2 x^{2}+3 x+1 ; g^{\prime}(x)=x^{2}-4 x+3 ; g^{\prime \prime}(x)=2 x-4 ; x=1$;

E-L $x=3 ; x=0 ; x=2 ; x=4$; intervalos de crecimiento: $(-\infty, 1) \boldsymbol{y}(3,+\infty)$; intervalos de decrecimiento: $(\mathbf{1}, 3) ; g(0)=3>0 ; g(2)=-1<0 ; g(4)=3>0 ; g^{\prime}(0)=3>0$; $g^{\prime}(2)=-1<0 ; g^{\prime}(4)=3>0$; punto máximo $\left(1, \frac{7}{3}\right)$ y mínimo $(3,1)$; intervalos de concavidad hacia abajo $(-\infty, 2)$ y hacia arriba $(2,+\infty) ; g^{\prime \prime}(1)=-2<0 ; g^{\prime \prime}(3)=2>0$; puntos de corte $(1,0)$ y $(3,0)$; punto de inflexión: $\left(2, \frac{5}{3}\right)$.

Tabular: ver Figura 6. 
Figura 6: Tablas de valores para gráficar a $\boldsymbol{g}$ y $\boldsymbol{g}^{\prime}$

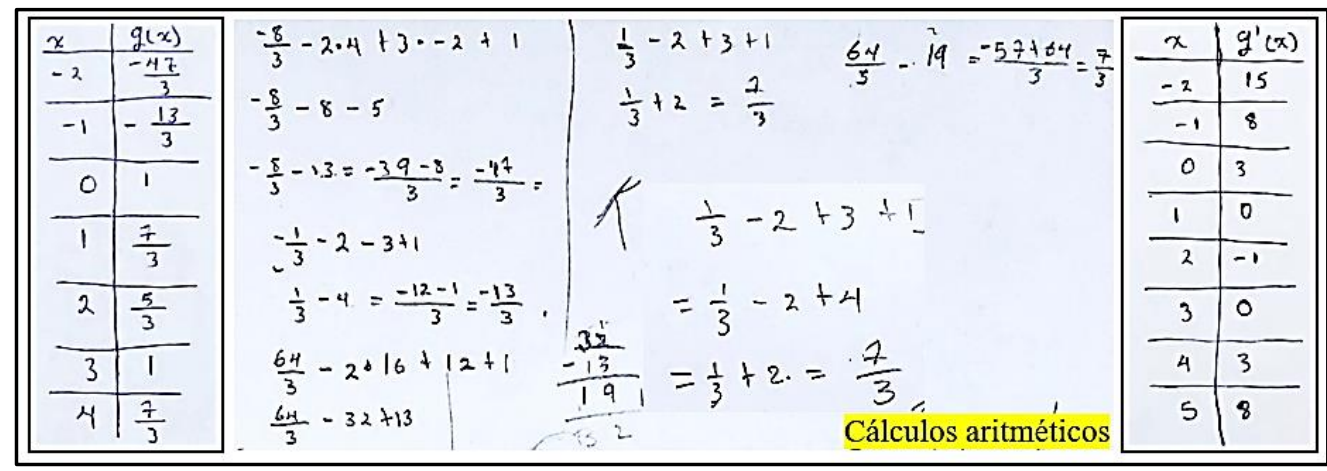

Fuente: Elaboración propia

Gráfico: ver Figura 7.

Figura 7: La gráfica de $\boldsymbol{g}$ y la gráfica de $\boldsymbol{g}^{\prime}$

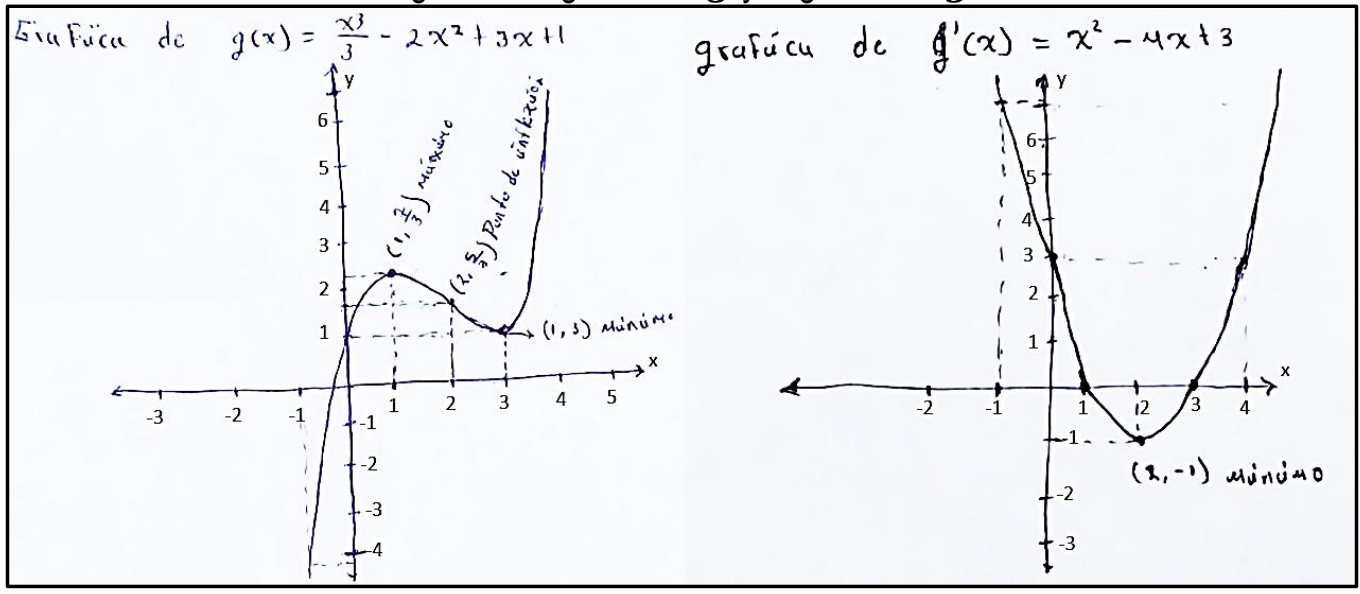

Fuente: Elaboración propia

Conceptos previos: punto, punto máximo o mínimo, punto de inflexión, punto crítico, recta, gráfica, ecuación, recta tangente, derivada, segunda derivada, derivada en un punto, punto de corte con un eje.

D D1: La derivada es la pendiente de la recta tangente a la curva en un punto.

D2: Un número crítico de $g$ es un número $c$ en el dominio de $g$ tal que $g^{\prime}(c)=0 \circ g^{\prime}(c)=0$ no existe D3: Sea $g$ continua en $c$. Llamamos a $(c, g(c))$ un punto de inflexión de la gráfica de $g$, si $g$ es cóncava hacia arriba a un lado de $c$ y cóncava hacia abajo del otro lado de $c$ (PURCELL, VARBERG y RIGDON, 2007).

Proposiciones previas: propiedad conmutativa.

Pr1: Los puntos críticos están en $x=1$ y $x=3$.

Pr2: Intervalos de crecimiento son: $(-\infty, \mathbf{1}) \boldsymbol{y}(3,+\infty)$ y el intervalo de decrecimiento es:

$(1,3)$.

Pr Pr3: El punto máximo es $\left(1, \frac{7}{3}\right)$ y el punto mínimo es $(3,1)$.

Pr4: El punto de inflexión es $\left(2, \frac{5}{3}\right)$.

Pr5: $g$ es cóncava hacia abajo en $(-\infty, 2)$ y cóncava hacia arriba en $(2,+\infty)$.

Pr6: Las funciones $g$ y $g^{\prime}$ son continuas.

Pr7: La derivada es una función de grado menor que la función original.

Procedimiento principal 1 (Pcp1): Determinación de los intervalos en los que $g$ es creciente 0 decreciente.

Pc Procedimientos auxiliares (Pca1.1): Calcular la derivada de una función de tercer grado

(Figura 10). 
Figura 10: Procedimiento para calcular el punto de inflexión

c) Para cncontras el punto de inflexion utrilizumos la segnda
deruivadu e igualumos a caro y resolvemos la ecuacuion
$g^{\prime \prime}(x)=2 x-4$
$2 x-4=0$
$x=2$
$g(2)=\frac{2^{3}}{3}-2(2)^{2}+3(2)+1=\frac{8}{3}-8+6+1=\frac{8}{3}-1=\frac{5}{3}$
$\therefore$ el punto $\left(2, \frac{5}{3}\right)$ es el punto de inflexion.

Fuente: Elaboración propia

Pcp4: Determinar los intervalos de concavidad de la función.

Pca4.1: Aplicar el criterio de la segunda derivada (ver Figura 11).

Pca4.2: Evaluar a $g$ en dos puntos ubicados antes y después del punto de inflexión (Figura 11).

Figura 11: Procedimiento para determinar los puntos de concavidad

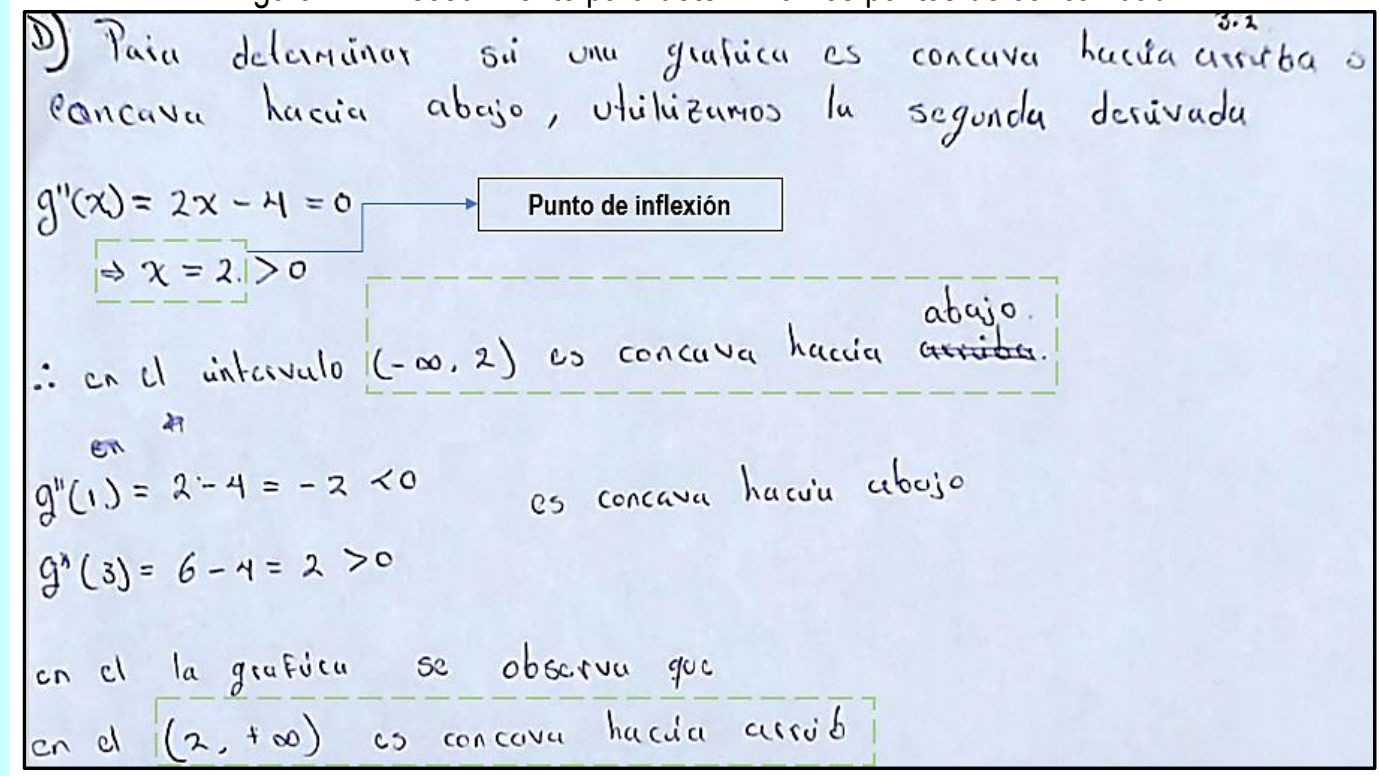

Fuente: Elaboración propia

Pcp5: Representación gráfica de la función $g$ y la de su derivada $g$ '.

Pca5.1: Representación gráfica de $g$ a partir de la expresión algebraica y una tabla de valores (ver Figuras 6 y 7 ).

Pca5.2: Representación gráfica de $g^{\prime}$ a partir de la expresión algebraica y una tabla de valores (ver Figuras 6 y 7 ).

Argumento 1 (A1): Tesis: Estos son los intervalos de crecimiento $(-\infty, \mathbf{1}) \boldsymbol{y}(\mathbf{3},+\infty)$ y el intervalo de decrecimiento es: $(\mathbf{1}, \mathbf{3})$.

Razón 1 (R1): Si una función es continua, los puntos críticos separan intervalos de crecimiento y/o decrecimiento.

A R2: En $g$, los puntos críticos son $x=1$ y $x=3$ porque para estos valores la derivada vale 0 .

R3: Existen tres intervalos y el signo de la derivada en cada uno siempre es el mismo. Si el signo es negativo es decreciente, y si el signo es positivo es creciente.

R4: Basta buscar el signo de la derivada para un valor cualquiera del intervalo. Para $x=0$ la derivada es positiva, por tanto, en el intervalo $(-\infty, 1) g$ es creciente. Para el valor $x=2$ la 
derivada es negativa, por tanto, en el intervalo $(1,3) g$ es decreciente. Para $x=4$ la derivada es positiva, entonces en el intervalo $(3,+\infty) g$ es creciente.

Conclusión 1: $g$ es creciente en los intervalos $(-\infty, 1)$ y $(3,+\infty)$ y decreciente en el intervalo $(1,3)$.

A2: Tesis: Este es el punto máximo $\left(1, \frac{7}{3}\right)$ y este es el punto mínimo $(3,1)$.

R1: En el intervalo $(-\infty, 1)$ la función es creciente y en el intervalo $(1,3)$ la función es decreciente, porque para un valor anterior a $x=1$ la derivada es positiva y para un valor después de $x=1$, la derivada es negativa, por tanto, $g$ tiene un máximo en $x=1$. Luego, en el intervalo $(3,+\infty)$ la función es creciente dado que, para un valor en este intervalo la derivada es positiva, entonces $g$ tiene un mínimo en $x=3$.

R2: La imagen de $x=1$ es $g(1)=\frac{7}{3}$ y la imagen de $x=3$ es $g(3)=1$.

Conclusión 2: El punto máximo es $\left(1, \frac{7}{3}\right)$ y el mínimo es $(3,1)$.

A3: Tesis: El punto de inflexión de la función es $\left(2, \frac{5}{3}\right)$.

R1: Si una función es continua, los puntos de inflexión separan intervalos de concavidad (cóncava hacia arriba a cóncava hacia abajo o viceversa).

R2: $g$ tiene un punto de inflexión en $x=2$ porque la segunda derivada $g$ " en ese valor es igual a 0 .

R3: Encuentra la imagen de $x=2$ que es $g(2)=\frac{5}{3}$.

Conclusión 3: $\left(2, \frac{5}{3}\right)$ si es el punto de inflexión.

A4: Tesis: $g$ es cóncava hacia abajo en $(-\infty, 2)$ y cóncava hacia arriba en $(2,+\infty)$.

R1: El punto de inflexión es $\left(2, \frac{5}{3}\right)$, existen dos intervalos y el signo de la segunda derivada en cada uno siempre es el mismo.

R2: Basta buscar el signo de la segunda derivada para un valor cualquiera del intervalo. Para $x=$ 1 la segunda derivada es negativa, por tanto, en el intervalo $(-\infty, 2) g$ es cóncava hacia abajo. Para el valor $x=3$ la segunda derivada es positiva, por tanto, en el intervalo $(2,+\infty), g$ es cóncava hacia arriba.

Conclusión 4: $g$ es cóncava hacia abajo en $(-\infty, 2)$ y cóncava hacia arriba en $(2,+\infty)$.

\section{Fuente: Elaboración propia.}

\subsection{Funciones semióticas (FS)}

A continuación, en la Figura 12 se presentan algunas funciones semióticas. 
Figura 12: Funciones semióticas establecidas con los objetos primarios por parte de E1

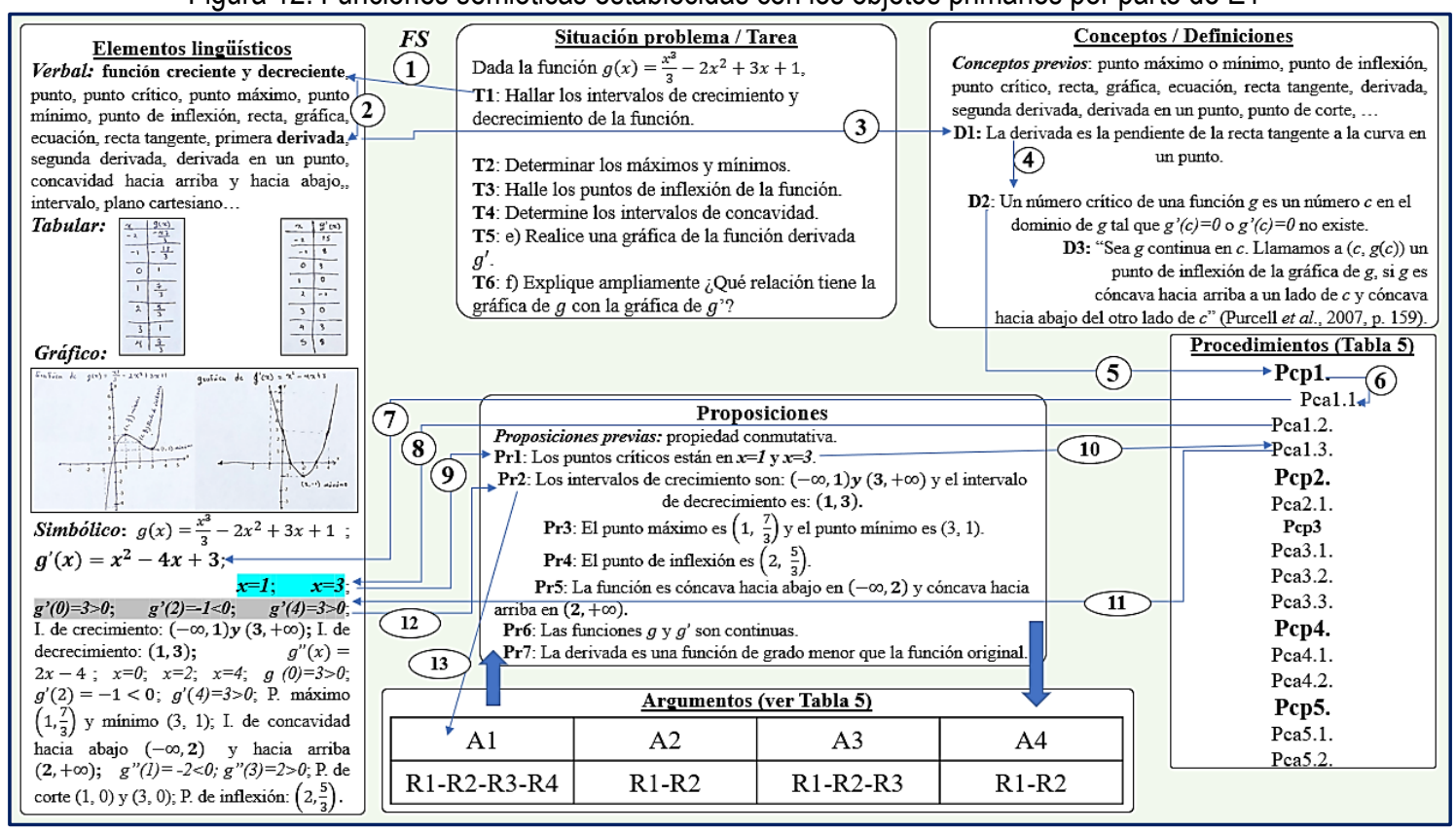

Fuente: Elaboración propia

En la Tabla 6 se presentan algunos casos de conexiones matemáticas establecidas por E1 al resolver la tarea (Figura 12), teniendo en cuenta el conglomerado de prácticas, procesos, objetos y funciones semióticas que constituyen a la conexión.

Tabla 6: Análisis detallado de la actividad matemática de E1 al resolver la tarea 3

\begin{tabular}{|c|c|c|c|c|}
\hline $\mathrm{Pm}$ & Procesos & Objetos & $\begin{array}{l}\text { Funciones } \\
\text { semióticas }\end{array}$ & $\begin{array}{l}\text { Conexión } \\
\text { matemática }\end{array}$ \\
\hline \multirow[b]{2}{*}{ Pm1 } & \multirow{2}{*}{$\begin{array}{l}\text { Significación / } \\
\text { comprensión. } \\
\text { Problematización. } \\
\text {-Resolución de } \\
\text { problemas- } \\
\text { Enunciación }\end{array}$} & $\begin{array}{l}\text { Tarea (T1) } \\
\text { D1: La derivada es la pendiente de la recta } \\
\text { tangente a la curva en un punto. }\end{array}$ & \multirow{2}{*}{$\begin{array}{l}\text { FS1 } \\
\text { FS2, FS3 y } \\
\text { FS4 }\end{array}$} & \\
\hline & & $\begin{array}{l}\text { D2: Un número crítico de una función } g \text { es un } \\
\text { número } c \text { en el dominio de } g \text { tal que } g^{\prime}(c)= \\
0 \text {. }\end{array}$ & & Significado \\
\hline Pm2 & $\begin{array}{l}\text { Resolución de } \\
\text { problemas } \\
\text { Representación } \\
\text { (verbal y } \\
\text { simbólica) }\end{array}$ & $\begin{array}{l}\text { Pca1.1: E1 calcula la derivada de } g \text {, que es } \\
\text { igual a } g^{\prime}(x)=x^{2}-4 x+3, \text { usando } \\
\text { implícitamente la fórmula } \frac{d\left(x^{n}\right)}{d x}=n x^{n-1} \text {. }\end{array}$ & $\begin{array}{l}\text { FS5, FS6, } \\
\text { FS7 }\end{array}$ & Procedimental \\
\hline Pm3 & $\begin{array}{l}\text { Resolución de } \\
\text { problemas } \\
\text { Representación } \\
\text { (verbal y } \\
\text { simbólica) }\end{array}$ & $\begin{array}{l}\text { Pca1.2: Calculó los puntos críticos de la } \\
\text { función. Usó el criterio de la primera derivada, } \\
\text { igualó a cero la derivada }\left(x^{2}-4 x+3=\right. \\
0) \text {, completó cuadrado y encontró los puntos } \\
\text { críticos }(x=1 \text { y } x=3) \text {. }\end{array}$ & FS8 y FS9 & Procedimental \\
\hline Pm4 & $\begin{array}{l}\text { Resolución de } \\
\text { problemas } \\
\text { Representación }\end{array}$ & $\begin{array}{l}\text { Pca1.3: Determinó los intervalos de } \\
\text { crecimiento }(-\infty, \mathbf{1}) \boldsymbol{y}(\mathbf{3},+\infty) \text { y } \\
\text { decrecimiento }(\mathbf{1}, \mathbf{3}) \text {. Usó el criterio de la } \\
\text { primera derivada y sustituyó } x=0 \text { en } g \text { y obtuvo } \\
g^{\prime}(0)=3>0 \text {, luego, sustituyó } x=2 \text { en } g \\
\text { y obtuvo } g^{\prime}(2)=-1<0, \text { y, por último, }\end{array}$ & $\begin{array}{c}\text { FS10 y } \\
\text { FS11 }\end{array}$ & Procedimental \\
\hline
\end{tabular}




\begin{tabular}{cllll}
\hline \multicolumn{5}{c}{$\begin{array}{l}\text { sustituyó } x=4 \text { en } g \text { y obtuvo } g^{\prime}(4)=3> \\
0 .\end{array}$} \\
$\begin{array}{c}\text { Argumentación } \\
\text { Enunciación } \\
\text { Representación }\end{array}$ & $\begin{array}{l}\text { Afirmó que en }(-\infty, \mathbf{1}) \text { la función es } \\
\text { creciente. Enunció que, en }(\mathbf{1}, \mathbf{3}) \text { la función } \\
\text { es decreciente. Enunció que, en }(\mathbf{3},+\infty) \text { la } \\
\text { función es creciente. }\end{array}$ & FS13 y & Implicación \\
\hline$\ldots$ & $\ldots$ & $\ldots$ & $\ldots$ & \\
\hline
\end{tabular}

Fuente: Elaboración propia

Por otra parte, el estudiante E2 tuvo dificultades para resolver la tarea, dado que, no estableció conexiones clave o necesarias soportadas a partir de prácticas (Pm diferentes a las de E1), procesos, objetos y funciones semióticas. En este contexto, E2 primero leyó la tarea y al parecer la entendió (Pm1). Segundo, halló la derivada de $g$ que le resultó $g^{\prime}(x)=x^{2}-4 x+3$ $(\mathrm{Pm} 2)$. Tercero, E2 encontró la segunda derivada $g^{\prime \prime}(x)=2 x-4(\mathrm{Pm} 3)$. Luego, igualó la derivada a cero obteniendo que el valor $x=2$ es el punto mínimo (Pm4). Seguidamente, evaluó la función $g$ en $x=2$, considerando que $y=-\frac{5}{3}$ es el máximo de la función (Pm5), y, por último, E2 dibujó una gráfica que no representa a la función y tampoco a su derivada (Pm6) (ver Figura 13).

Figura 13: Procedimiento realizado por E2

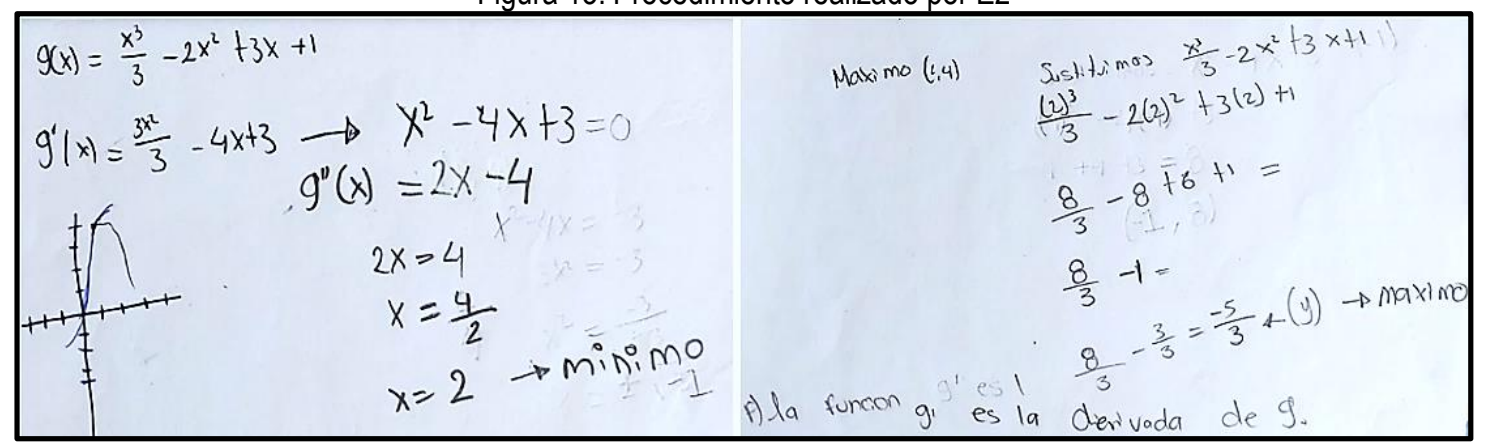

Fuente: Elaboración propia

\section{Discusión y reflexiones sobre la naturaleza de la comprensión matemática}

Para E1, se han analizado las conexiones matemáticas que establece cuando resuelve una tarea sobre la derivada enfatizando en las prácticas, procesos, objetos y funciones semióticas que los relacionan, que es una perspectiva de análisis detallado lograda en la articulación entre la TAC y el EOS (RODRÍGUEZ-NIETO et al., 2021). Asimismo, las conexiones matemáticas también informan acerca del tipo de comprensión sobre la derivada que tiene $\mathrm{E} 1$, dado que, sus respuestas fueron correctas teniendo en cuenta la actividad matemática y las conexiones que estableció, que, de hecho, son institucionales. A partir de las conexiones se infiere que, E1 tiene un tipo de comprensión de la derivada que le permite usarla de manera competente y argumentada para resolver tareas en un contexto gráfico. De esta manera se validan las afirmaciones de Berry y Nyman (2003); Cai y Ding (2015), García-García y Dolores-Flores (2019), NCTM (2000), entre 
otros, cuando revelan que, hacer conexiones matemáticas es un buen indicador para la comprensión de conceptos matemáticos.

Para el caso del estudiante E2 se desarrolló un proceso similar al descrito con E1 y, se realizó una comparación entre el análisis de la actividad matemática realizada por E1 y la realizada por E2. Esta comparación permite observar las prácticas matemáticas que faltan o la conexión incorrecta (conexión personal) que hacen los estudiantes; lo cual explica la causa de las dificultades que ha tenido para resolver correctamente la tarea. En la Figura 14, se presenta la producción escrita de E2 cuando resolvió la tarea, no obstante, en la Pm4 hizo una conexión incorrecta entre el valor donde la segunda derivada se hace cero y el punto mínimo. Luego, en Pm5 estableció otra conexión incorrecta entre el punto $\left(2,-\frac{5}{3}\right)$ y el punto máximo de la función. Por último, E2 conecta de forma incorrecta la función $g(x)=\frac{x^{3}}{3}-2 x^{2}+3 x+1$ y la gráfica (ver Figura 13). Estas conexiones incorrectas hacen que E2 no realice las otras Pm que son clave o indispensables para resolver la tarea de forma correcta. Asimismo, esta situación manifiesta que E1 tiene una comprensión, que podríamos decir de tipo instrumental, sobre el uso de la primera y segunda derivada, pero no llega a tener una comprensión, que podríamos llamar conceptual, que le permita esbozar la gráfica de la función y relacionarla con la gráfica de su derivada.

Ahora bien, lo más relevante es que hemos podido explicar la comprensión de los estudiantes con base en el establecimiento o no de determinadas conexiones, las cuales, a su vez, son una manera compactada de considerar una porción de actividad matemática que implica determinadas prácticas y procesos, donde intervienen objetos primarios que se relacionan por medio de funciones semióticas. Consideramos que este estudio podría ampliarse con una población mayor de participantes para geneneralizar la temática de la comprensión.

\section{Agradecimientos}

Este artículo es un producto del trabajo de tesis doctoral del Dr. Camilo Andrés RodríguezNieto apoyado por la Universidad Autónoma de Guerrero y CONACYT. Este artículo es parte del proyecto de investigación PGC2018-098603-B-I00 (MCIU/AEI/FEDER, UE).

\section{Referencias}

ARNON, Llana et al. APOS theory: a framework for research and curriculum development in mathematics education. New York: Springer-Verlag, 2014.

BERRY, John.; NYMAN, Melvin. Promoting students' graphical understanding of the calculus. The Journal of Mathematical Behavior, v. 22, n. 4, p. 479-495, 2003. 
BORJI, Vahid.; RADMEHR, Farzad.; FONT, Vicenç. The impact of procedural and conceptual teaching on students' mathematical performance over time, International Journal of Mathematical Education in Science and Technology, v. 52, n.3, p. 404-426, 2021.

BORJI, Vahid., et al. Application of the Complementarities of Two Theories, APOS and OSA, for the Analysis of the University Students' Understanding on the Graph of the Function and its Derivative. EURASIA Journal of Mathematics, Science and Technology Education, v. 14, n. 6 , p. 2301-2315, 2018.

BRAUN, Virginia.; CLARKE, Victoria. Using thematic analysis in psychology. Qualitative Research in Psychology, v. 3, n. 2, p. 77-101, 2006.

BUSINSKAS, Aldona Monika. Conversations about connections: How secondary mathematics teachers conceptualize and contend with mathematical connections. 2008. 183f. Disertación (PhD of Philosophy) - Faculty of Education, Simon Fraser University. Canada.

CAI, Jinfa.; DING, Meixia. On mathematical understanding: perspectives of experienced Chinese mathematics teachers. Journal of Mathematics Teacher Education, v. 20, n. 1, p. 5-29. 2015.

CHIGEZA, Philemon. Translating between and within representations: mathematics as lived experiences and interactions. In V. Steinle, L. Ball, \& C. Bardini (Eds.), Mathematics Education: Yesterday, today and tomorrow. Proceedings of the $36^{\text {th }}$ annual conference of the mathematics education research group of Australasia (pp. 178-185). 2013. Melbourne, VIV: MERGA.

COHEN, Louis.; MANION, Lawrence.; MORRISON, Keith. Research methods in education. London and New York: Routledge, 2018.

DOLORES-FLORES, Crisologo.; GARCÍA-GARCÍA, Javier. Conexiones Intramatemáticas y Extramatemáticas que se producen al Resolver Problemas de Cálculo en Contexto: un Estudio de Casos en el Nivel Superior. Bolema, Boletim de Educação Matemática, v. 31, n. 57, p. 158-180, 2017. DOI: http://dx.doi.org/10.1590/1980-4415v31n57a08

DOLORES-FLORES, Crisologo.; RIVERA-LÓPEZ, Martha Iris.; GARCÍA-GARCÍA, Javier. Exploring mathematical connections of pre-university students through tasks involving rates of change. International Journal of Mathematics Education in Science and Technology, v. 50, n. 3, p. 369-389, 2019. http://dx.doi.org/10.1080/0020739X.2018.1507050

LOBO, Rogério dos Santos. Utilizando a Derivada para inferir sobre a velocidade de contaminação do novo Coronavírus: uma possibilidade para as aulas de Cálculo. Revemop, v. 2, n. e202021, p. 1-18, 2020. DOI: https://doi.org/10.33532/revemop.e202021

ELI, Jennifer.; MOHR-SCHROEDER, Margaret. J.; LEE, Carl. Exploring mathematical connections of prospective middle-grades teachers through card-sorting tasks. Mathematics Education Research Journal, v. 23, n. 3, p. 297-319. 2011.

ELI, Jennifer.; MOHR-SCHROEDER, Margaret. J.; LEE, Carl. Mathematical connections and their relationship to mathematics knowledge for teaching geometry. School Science and Mathematics, v. 113, n. 3, p. 120-134. 2013. 
EVITTS, Thomas. Investigating the mathematical connections that preservice teachers use and develop while solving problems from reform curricula. 2004 Disertación (PhD of Philosophy)). Pennsylvania State University-College of Education. EE. UU.

FÉLIX SANDOVAL, G. C.; MONTEVERDE, A. G. Taxa de variação instantânea com magnitudes infinitamente pequenas: uma experiência no Ensino à Distância. Revemop, v. 3, p. e202114. DOI: https://doi.org/10.33532/revemop.e202114

FERRINI-MUNDY, Joan.; GRAHAM, Karen. (1994). Research in calculus learning: Understanding of limits, derivatives, and integrals. MAA notes, p. 31-46. 1994.

FONT, Vicenç. Una perspectiva ontosemiótica sobre cuatro instrumentos de conocimiento que comparten un aire de familia: particular/general, representación, metáfora y contexto. Educación matemática, v.19, n. 2, p. 95-128. 2007.

FONT, Vicenç et al. Mathematical objects through the lens of two different theoretical perspectives: APOS and OSA. Educational Studies in Mathematics, v. 91, n. 1, p. 107-122, oct. 2016.

FONT, Vicenç.; CONTRERAS, Ángel. (2008). The problem of the particular and its relation to the general in mathematics education. Educational Studies in Mathematics, v. 69, p. 33-52. 2008.

FONT, Vicenç et al. The emergence of objects from mathematical practices. Educational Studies in Mathematics, v. 82, p. 97-124. 2013.

FUENTEALBA, Claudio.; BADILLO, Edelmira.; SÁNCHEZ-MATAMOROS, Gloria. Puntos de noderivabilidad de una función y su importancia en la comprensión del concepto de derivada. Educação e Pesquisa, v. 44, p. 1-20. 2018.

FUENTEALBA, Claudio et al. The understanding of the derivative concept in higher education. EURASIA Journal of Mathematics, Science and Technology Education, v. 15, n. 2, 1-15. 2018.

GARCÍA-GARCÍA, Javier. Escenarios de exploración de conexiones matemáticas. Números: Revista de didáctica de las matemáticas, v. 100, p. 129-133. 2019.

GARCÍA-GARCÍA, Javier.; DOLORES-FLORES, Crisologo. Intra-mathematical connections made by high school students in performing Calculus tasks. International Journal of Mathematical Education in Science and Technology, v. 49, n. 2, p. 227-252. 2018.

GARCÍA-GARCÍA, Javier.; DOLORES-FLORES, Crisologo. Pre-university students' mathematical connections when sketching the graph of derivative and antiderivative functions. Mathematics Education Research Journal, v. 33. p. 1-22. 2019. https://doi.org/10.1007/s13394-019-00286-x

GARCÍA-GARCÍA, Javier.; DOLORES-FLORES, Crisologo. Exploring pre-university students' mathematical connections when solving Calculus application problems. International Journal of Mathematical Education in Science and Technology, 2020. DOI: 10.1080/0020739X.2020.1729429

GODINO, Juan.; BATANERO, Carmen. Significado institucional y personal de los objetos matemáticos. Recherches en didactique des Mathématiques, v. 4, n.3, p. 325-355, 1994. 
GODINO, Juan et al. Una perspectiva ontosemiótica de los problemas y métodos de investigación en educación matemática. Revemop, v. 3, n. e202107, p. 1-30, 2021. DOl: https://doi.org/10.33532/revemop.e202107

GODINO, Juan et al. The onto-semiotic approach to research in mathematics education. ZDM The International Journal on Mathematics Education, v. 39, n. 1-2, p. 127-135. 2007.

GODINO, Juan et al. The onto-semiotic approach: implications for the prescriptive character of didactics. For the Learning of Mathematics, v. 39, n. 1, p. 37- 42. 2019.

GODINO, Juan et al. Why is the learning of elementary arithmetic concepts difficult? Semiotic tools for understanding the nature of mathematical objects. Educational Studies in Mathematics, v. 77, n. 2, p. 247-265. 2010.

GREENO, James. Indefinite goals in well-structured problems. Psychological Review, v.83, n.6, p. 479-491. 1976.

HIEBERT, James.; CARPENTER, Thomas. Learning and teaching with understanding. In: Grouws D. A. (Ed.). Handbook of research of mathematics teaching and learning. New York: Macmillan, 1992, p. 65-79.

HIEBERT, James.; LEFEVRE, Patricia. Conceptual and procedural knowledge in mathematics: An introductory analysis. In J. Hiebert (Ed.), Conceptual and procedural knowledge: The case of mathematics, Hillsdale, NJ: Lawrence Erlbaum Associates, Inc. 1986, p. 1-27.

INSTITUTO NACIONAL DE FORMACIÓN DOCENTE (INFD). Proyecto de mejora para la formación inicial de profesores para el nivel secundario. Área: Matemática. Ministerio de Educación, Instituto Nacional de Formación Docente y Secretaría de Políticas Universitarias. Buenos Aires, 2010. Disponible en: https://cedoc.infd.edu.ar/upload/Matematica.pdf. Acceso: 03 may. 2019.

KASTBERG, Signe. Understanding mathematical concepts: The case of the logarithmic function. 2002. 2009f. Disertación (PhD of Philosophy) - Dean of the Graduate School, University of Georgia. Georgia.

KENEDI, Ary Kiswanto; et al. Mathematical connection of elementary school students to solve mathematical problems. Journal on Mathematics Education, v. 10, n. 1, p. 69-80. 2019.

KULA-ÜNVER, Semiha. How do pre-service mathematics teachers respond to students' unexpected questions related to the second derivative?. Journal of Pedagogical Research, v.4, n.3, p. 359-374. 2020.

LUGO-ARMENTA, J. G.; PINO-FAN, L. R.; RUIZ HERNANDEZ, B. R. Significados de Referencia del Estadístico Chi-cuadrada: una Mirada Histórico-epistemológica. Revemop, v. 3, p. e202108, 21 jun. 2021. DOl: https://doi.org/10.33532/revemop.e202108

MEEL, David. Modelos y teorías de la comprensión matemática: Comparación de los modelos de Pirie y Kieren sobre el crecimiento de la comprensión matemática y la Teoría APOE. Revista Latinoamericana de Investigación en Matemática Educativa, RELIME, v.6, n.3, p. 221-278. 2003. 
MHLOLO, Michael. Mathematical connections of a higher cognitive level: A tool we may use to identify these in practice. African Journal of Research in Mathematics, Science and Technology Education, v. 16, n. 2, p. 176-191. 2012.

MHLOLO, Michael.; VENKAT, Hamsa.; SCHÄFER, Marc. The nature and quality of the mathematical connections teachers make. Pythagoras, v. 33, n. 1, p. 1-9. 2012.

MICHENER, Edwina. Understanding understanding Mathematics. Cognitive Science, v. 2, p. 361383, 1978.

NATIONAL COUNCIL OF TEACHERS OF MATHEMATICS (NCTM). Principles and standards for school mathematics. Reston, VA: NCTM, 2000.

NATIONAL RESEARCH COUNCIL (NRC). Adding it up: Helping children learn mathematics. J. Kilpatrick, J. Swafford, and B. Findell (Eds.). Mathematics Learning Study Committee, Center for Education, Division of Behavioral and Social Sciences and Education. Washington, DC: National Academy Press. 2001.

NEMIROVSKY, Ricardo.; RUBIN, Andee. Students' tendency to assume resemblances between a function and its derivatives. TERC Working 2-92. Cambridge MA: TERC.1992.

NICKERSON, Raymond. S. Understanding Understanding. American Journal of Education, v.93, n.2, p. 201-239, 1985.

OEHRTMAN, Michael.; CARLSON, Marilyn.; THOMPSON, Patrick. Foundational reasoning abilities that promote coherence in students' function understanding. In Marilyn Carlson \& Chris Rasmussen (Eds.). Making the connection: Research and practice in undergraduate mathematics, MAA Notes Volume, 73, p. 27-41. Washington, DC: Mathematical Association of America. 2009.

ORTIZ, Juan; FONT, Vicenç. Significados personales de los futuros profesores de educación primaria sobre la media aritmética. Educación Matemática, v. 23, no 2, p. 91-109. 2011.

PAMBUDI, Didik et al. Mathematical connection profile of junior high school students in solving mathematical problems based on gender difference. International Journal of Scientific Research and Management, v.6, n. 08, p. 73-78. 2018.

PINO-FAN, Luis.; GODINO, Juan.; FONT, Vicenç. (2011). Faceta epistémica del conocimiento didáctico-matemático sobre la derivada. Educação Matemática Pesquisa, v.13, n.1, p. 141-178. 2011.

PINO-FAN, Luis.; GODINO, Juan.; FONT, Vicenç. Una propuesta para el análisis de las prácticas matemáticas de futuros profesores sobre derivadas. Bolema, Boletim de Educação Matemática, v. 29 , n. 51 , p. 60-89. 2015.

PINO-FAN, Luis.; GODINO, Juan.; FONT, Vicenç. Assessing key epistemic features of didactic mathematical knowledge of prospective teachers: the case of the derivative. Journal of Mathematics Teacher Education, v. 21, n. 1, p. 63-94. 2018.

PINO-FAN, Luis.; GUZMÁN, Ismenia.; FONT, Vicenç.; DUVAL, Raymond. Analysis of the underlying cognitive activity in the resolution of a task on derivability of the absolute-value function: Two theoretical perspectives. PNA, v. 11, n. 2, p. 97-124. 2017. 
PIRIE, S., \& KIEREN, T. A recursive theory of mathematical understanding. For the learning of mathematics, 9(3), p. 7-11, 1989.

PIRIE, Susan.; KIEREN, Thomas. Growth in mathematical understading: how can we characterise it and how can we represent it? Educational Studies in Mathematics, v. 26, p. 165-190. 1994.

POLYA, George. Mathematical Discovery, John Wiley \& Sons. 1962.

POLYA, George. Cómo plantear y resolver problemas. México: Editorial Trillas. 1989.

PURCELL, Edwin.; VARBERG, Dale.; RIGDON, Steven. Cálculo diferencial e integral. Pearson. 2007.

RODRÍGUEZ-NIETO, Camilo Andrés. Explorando las conexiones entre sistemas de medidas usados en prácticas cotidianas en el municipio de Baranoa. IE Revista de Investigación Educativa de la REDIECH, v. 11, n. e-857, p. 1-30. 2020.

RODRÍGUEZ-NIETO, Camilo Andrés. Conexiones etnomatemáticas entre conceptos geométricos en la elaboración de las tortillas de Chilpancingo, México. Revista de investigación desarrollo e innovación, v. 11, n. 2, p. 273-296. 2021.

RODRÍGUEZ-NIETO, Camilo Andrés; et al. Mathematical connections from a networking theory between Extended Theory of Mathematical connections and Onto-semiotic Approach. International Journal of Mathematical Education in Science and Technology. 2021. https://doi.org/10.1080/0020739X.2021.1875071

RODRÍGUEZ-NIETO, Camilo Andrés.; RODRÍGUEZ-VÁSQUEZ, Flor Monserrat.; FONT, Vicenç. A new view about connections. The mathematical connections established by a teacher when teaching the derivative. International Journal of Mathematical Education in Science and Technology. 2020. https://doi.org/10.1080/0020739X.2020.1799254

RODRÍGUEZ-NIETO, Camilo Andrés.; RODRÍGUEZ-VÁSQUEZ, Flor Monserrat.; GARCÍAGARCÍA, Javier. Pre-service mathematics teachers' mathematical connections in the context of problem-solving about the derivative. Turkish Journal of Computer and Mathematics Education, v. 12, n. 1, p. 202-220. 2021.

RODRÍGUEZ-VÁSQUEZ, Flor Monserrat.; ARENAS-PEÑALOZA, Jhonatan Categories to assess the understanding of university students about a mathematical concept. Acta Scientiae, v. 23, n. 1, p. 102-134. 2021.

SÁNCHEZ-MATAMOROS, Gloria; GARCÍA, Mercedes.; LLINARES, Salvador. La comprensión de la derivada como objeto de investigación en didáctica de la matemática. Revista latinoamericana de investigación en matemática educativa, v. 11, n. 2, p. 267-296. 2008.

SIERPINSKA, Anna. Some Remarks on Understanding in Mathematics. For the Learning of Mathematics, v.10, n. 3, p. 24-36. 1990.

SKEMP, Richard. Relational understanding and instrumental understanding. Mathematics teaching, v. 77, n. 1, p. 20-26. 1976.

SKEMP, Richard. Relational understanding and instrumental understanding', Arithmetic Teacher, v. 26, n. 3, p. 9-15. 1978. 
SKEMP, Richard. (1980). Psicología del aprendizaje de las Matemáticas. Madrid: Ediciones Morata S.A

UBUZ, Behiye. First year engineering students' learning of point of tangency, numerical calculation of gradients, and the approximate value of a function at a point through computers. Journal of Computers in Mathematics and Science Teaching, v. 20, n. 1, p. 113-137. 2001.

VERÓN, Manuel Alejandro; GIACOMONE, Belén. Análisis de los significados del concepto de diferencial desde una perspectiva ontosemiótica. Revemop, v. 3, n. e202109, p. 1-27, 2021. DOI: https://doi.org/10.33532/revemop.e202109

VOSKOGLOU, Michael. Application of grey numbers to assessment of the understanding the graphical representation of the derivative. American Journal of Educational Research, v. 5, n. 11, p. 1167-1171. 2017. doi: 10.12691/education-5-11-9.

YAVUZ-MUMCU, Hayal. Matematiksel ilişkilendirme becerisinin kuramsal boyutta incelenmesi: türev kavramı örneği. Turkish Journal of Computer and Mathematics Education, v. 9, n. 2, p. 211-248. 2018. DOI: 10.16949/turkbilmat.379891. 